\title{
ACTIONS OF THE TORUS ON 4-MANIFOLDS. I
}

\author{
BY \\ PETER ORLIK AND FRANK RAYMOND( ${ }^{(1)}$ \\ Dedicated to the memory of Edward Halpern
}

\begin{abstract}
Smooth actions of the 2-dimensional torus group $S O(2) \times S O(2)$ on smooth, closed, orientable 4-manifolds are studied. A cross-sectioning theorem for actions without finite nontrivial isotropy groups and with either fixed points or orbits with isotropy group isomorphic to $S O(2)$ yields an equivariant classification for these cases. This classification is made numerically specific in terms of orbit invariants. A topological classification is obtained for actions on simply connected 4-manifolds. It is shown that such a manifold is an equivariant connected sum of copies of complex projective space $C P^{2},-C P^{2}$ (reversed orientation), $S^{2} \times S^{2}$ and the other oriented $S^{2}$ bundle over $S^{2}$. The latter is diffeomorphic (but not always equivariantly diffeomorphic) to $C P^{2} \#-C P^{2}$. The connected sum decomposition is not unique. Topological actions on topological manifolds are shown to reduce to the smooth case. In an appendix certain results are extended to torus actions on orientable 4dimensional cohomology manifolds.
\end{abstract}

Introduction. In this article we begin the study of actions of the two-dimensional torus group $S O(2) \times S O(2)$ on 4-manifolds. Our chief motivation for this study is that it enables one to construct, in a systematic way, a large number of nicely behaved 4-manifolds. These 4-manifolds can be described in terms of certain weighted 2-manifolds (orbit spaces with their orbit structures). To write down all possible actions of the torus on 4-manifolds is not too difficult but it is entirely a different matter to characterize topologically the 4-manifold from the orbit data.

We may think of this study as two separate problems. By the equivariant problem we mean being able to write down enough data (orbit space, orbit types, etc.) so that when two sets of data arising from two actions (not necessarily on the same manifold) are examined, one can decide whether or not there is a homeomorphism between the two manifolds which is equivariant with respect to both actions. This problem is considerably easier than the topological classification problem.

By topological classification we mean the topological identification and classification of the manifolds arising in terms of their data used for deciding the first problem.

Received by the editors December 15, 1969.

AMS 1968 subject classifications. Primary 5560, 5747.

Key words and phrases. Group action, 4-manifold, torus, orbit space, cross-section, isotropy groups, equivariant map, equivariant connected sum.

$\left.{ }^{1}\right)$ The authors were supported in part by NSF.

Copyright (C) 1970, American Mathematical Society 
As an illustration of this approach the first problem for actions of $S O(2)$ on 3dimensional manifolds is solved in [8]. The second problem is also solved there when the action has fixed points. In terms of the orbit data it is shown when the manifold is compact (and closed) that it is a certain equivariant connected sum of lens spaces (including the sphere and $S^{2} \times S^{1}$ ) and in the nonorientable case some additional sums of nonorientable handles and $R P^{2} \times S^{1}$ 's. In [5], this topological classification is given for the closed case without fixed points ("Seifert manifolds"). Here, except for certain exceptional cases, these 3-manifolds are $K(\pi, 1)$ 's, have a unique $S O(2)$-action, and a manifold is determined by its fundamental group which, in turn, is calculated from orbit information.

Both of these problems are solved here for torus actions on closed oriented simply connected 4-manifolds. We prove that the 4-manifold is a specific equivariant connected sum of the 4-sphere, oriented complex projective spaces, and handles $\left(S^{2} \times S^{2}\right.$ 's) (§5). In addition, the equivariant problem is solved if finite stability groups other than the identity are absent ( $\$ 1,4$ and appendix).

Crucial to the solution of these problems and to this entire paper is the crosssectioning theorem proved in $\S 1$. In $\S 2$ topological torus actions on lens spaces are classified. $\$ 3$ studies the (topological) normal bundles of certain embedded 2-spheres in terms of orbit data. In particular, the 4-manifold is constructible by certain "plumbing" operations along these embedded 2-spheres and other "glueing" operations.

In $\$ 4$ we give a precise form (in terms of numerical invariants) of our solution to the equivariant classification problem.

We have phrased most of this paper in the context of smooth actions on smooth manifolds. This is really only a convenience since all topological actions are topologically equivalent to smooth ones and smooth classification is equivalent to topological classification in this context. We have relegated to an appendix the proof of some of these facts. The arguments in the topological or cohomological case are all harder than in the smooth case since one is trying to obtain the same conclusions from much weaker hypotheses. In fact, in the appendix we treat toral actions on sufficiently general 4-dimensional cohomology manifolds to include all topological manifolds and certain types of singular complex varieties, e.g. Brieskorn varieties, which admit a toral action. This is done with really not much extra pain and is very natural in the context in which we are working.

Illustrations and examples that go beyond our goal in $\$ 5$ are given in the text. In particular, one finds in (5.8) a simple argument that $C P^{2} \#\left(-C P^{2}\right) \# C P^{2}$ is diffeomorphic to $\left(S^{2} \times S^{2}\right)$ \#CP $P^{2}$, see [3].

Another example (5.10) shows that a toral action on $C P^{2} \#\left(-C P^{2}\right)$ may possess no equivariant 3 -sphere which realizes the splitting into connected sums. In the appendix, an algebraic action of the torus is described on a singular Brieskorn variety which topologically, in this case, is a cone over a lens space. 
In sequels we intend to complete the equivariant problem and continue the topological classification. In particular, we have a stable topological classification in the closed oriented nonsimply connected case. Very precise information is also available when all orbits are two dimensional and the genus of the orbit space is greater than or equal to 1 .

Added in proof. We would like to call attention to G. E. Bredon, On a certain class of transformation groups, Michigan Math. J. 9 (1962), 385-393. It contains, as a special case, the analysis of the orbit structure and a local cross-sectioning theorem in the neighborhood of a fixed point for toral actions on an integral $4-\mathrm{cm}$. Since we wished to obtain very specific global results, the overlap with $\S 1$ is slight.

1. Orbit structure. In $\$ \$ 1$ through 5 we will be mainly concerned with smooth actions of the torus $G=T^{2}=S O(2) \times S O(2)$ on closed smooth 4-manifolds. However, most of our results are valid in the topological category. The needed changes in the arguments together with an exploration of results in the cohomological framework with simple examples of actions on singular complex algebraic varieties will be discussed in the appendix. Henceforth, unless specified otherwise, we shall tacitly assume that $G$ is acting smoothly on a smooth manifold.

1.1. Proposition. If $G$ acts effectively on a cohomology manifold $X$, then there is a principal orbit.

Let us parametrize $G$ by $(\varphi, \theta)$ where $0 \leqq \varphi<2 \pi, 0 \leqq \theta<2 \pi$. Define the subgroup $G(m, n)=\{(\varphi, \theta): m \varphi+n \theta=0,(m, n)=1\}$. Note that $G(m, n)$ is isomorphic to $S O(2)$.

The subgroups $G(m, n)$ and $G\left(m^{\prime}, n^{\prime}\right)$ together generate the homology of $G=T^{2}$ if and only if $\left|\begin{array}{cc}m & m^{\prime} \\ n & n^{\prime}\end{array}\right|= \pm 1$. However they generate $G$ as a group provided $\left|\begin{array}{cc}m & m^{\prime} \\ n & n^{\prime}\end{array}\right| \neq 0$.

1.2. Proposition. If $M$ is an orientable 4-manifold then $M / G(m, n)$ is a 3manifold with boundary.

1.3. Proposition. If $M$ is closed and orientable then the orbit space $M / G=M^{*}$ is a compact 2-manifold.

Both propositions follow immediately from the (differentiable) slice theorem [1, Chapter 8]. These propositions are considerably more difficult in the cohomological case.

1.4. The table below lists the possible isotropy groups together with the slice, the action of the isotropy group on the slice and the image of the orbit on $M^{*}$. Again recall that $M$ is orientable.

The details follow easily. The fact that an effective action around a fixed point 


\begin{tabular}{|c|c|c|c|c|}
\hline $\begin{array}{l}\text { isotropy } \\
\text { group }\end{array}$ & orbit & slice & $\begin{array}{c}\text { action of isotropy } \\
\text { group on slice }\end{array}$ & image of orbit on $M^{*}$ \\
\hline$Z_{n} \times e$ & $T^{2}$ & $D^{2}$ & rotation & isolated interior point \\
\hline$Z_{n} \times Z_{m}$ & $T^{2}$ & $D^{2}$ & rotation & $\begin{array}{l}\text { isolated interior point } \\
\text { need }(n, m)=1\end{array}$ \\
\hline$G(m, n)$ & $S^{1}$ & $D^{3}$ & rotation & boundary point \\
\hline$G(m, n) \times Z_{p}$ & \multicolumn{4}{|c|}{$\begin{array}{l}\text { not possible for } p \geqq 2 \text { since the orbit would be 1-dimensional with slice } D^{3} \text {, } \\
\text { but } S^{1} \times Z_{p} \notin S O(3)\end{array}$} \\
\hline$G$ & $\begin{array}{l}\text { fixed } \\
\text { point }\end{array}$ & $D^{4}$ & $\begin{array}{l}\text { rotation in two planes } \\
\text { by } G\left(m_{1}, n_{1}\right) \text { and } \\
G\left(m_{2}, n_{2}\right)\end{array}$ & $\begin{array}{l}\text { isolated boundary point } \\
G\left(m_{1}, n_{1}\right), G\left(m_{2}, n_{2}\right) \\
\text { need } \mid \begin{array}{ll}m_{1} & m_{2} \\
n_{1} & n_{2}\end{array}= \pm 1\end{array}$ \\
\hline
\end{tabular}

requires $\left|\begin{array}{ll}m_{1} & m_{2} \\ n_{1} & n_{2}\end{array}\right|= \pm 1$ will be seen in $\S 2$ from a different point of view. In the appendix the general nonmanifold case, $\left|\begin{array}{ll}m_{1} & m_{2} \\ n_{2} & n_{2}\end{array}\right| \neq 0$ will be considered.

Definition. An orbit with nontrivial finite stability group will be called an $E$ orbit. The totality of $E$-orbits is denoted by $E$. An orbit whose stability group is isomorphic to the circle group will be called a $C$-orbit and the totality of $C$-orbits is denoted by $C$. The totality of fixed points is denoted by $F$.

1.5. Next we turn our attention to the problem of finding a cross-section to the orbit map.

Let $X$ be a compact Hausdorff space with an effective $G$ action. Assume that the orbit space is $X^{*}=I \times I$ and that there are only two types of orbits. The points on the arc $0 \times I$ have stability group $G(m, n)$ with relatively prime $(m, n)$ and all other points correspond to principal orbits, hence, by (1.1) to $T^{2}$.

1.6. Lemma. The orbit map $\pi: X \rightarrow X^{*}$ has a cross-section. Moreover, any cross-section given on the arc $A=(I \times 1) \cup(1 \times I) \cup(I \times 0)$ may be extended to a cross-section over all of $X^{*}$.

Proof. For the given $(m, n)$ we can find $(p, q)$ such that $\left|\begin{array}{ll}p & m \\ q & n\end{array}\right|=1$. Then the action of $G(p, q)$ is free on $X$. Let $Y=X / G(p, q)$. The orbit map $\pi^{\prime}: X \rightarrow Y$ is a principal $G(p, q)$ bundle. The action of $G(m, n)$ on $Y$ is semifree and the fixed points of $\pi^{\prime \prime}: Y \rightarrow X^{*}$ map onto $0 \times I$. The action of $G(m, n)$ over $X^{*}-(0 \times I)$ is free and it is a product action. Any cross-section to this action can be trivially extended to all of $X^{*}$. Thus $Y$ is homeomorpinic to $X^{*} \times S^{1}$ with each circle $0 \times t \times S^{1}$ collapsed to a point, which is a 3-cell. The action of $G(m, n)$ is just rotation about the axis $(0 \times I)$. Now the free action of $G(p, q)$ over $Y$ must be trivial and hence $X=Y \times S^{1}$, with a cross-section. We combine the two cross-sections $X^{*} \rightarrow Y$ and $Y \rightarrow X$ to obtain the desired cross-section to $\pi$.

Now suppose that a cross-section is already given on $A$. This gives a section over $A$ for the orbit map $\pi$. Since $H^{2}\left(X^{*}-0 \times I, A-(0 \times 0 \cup 0 \times 1) ; Z\right)=0$ we can extend 
over $X^{*}$ any cross-section $\chi^{\prime \prime}: A \rightarrow Y$. Since $H^{2}\left(X^{*}, A ; Z\right)=0$ we can lift the section $\chi^{\prime \prime}$ to $X$ to extend any lift over $\chi^{\prime \prime}(A)$.

1.7. Lemma. Suppose that $\pi: X \rightarrow X^{*}$ is the orbit map of a G-action on a compact Hausdorff space and assume that $X^{*}=S^{1} \times I$, where $S^{1} \times 0$ corresponds to orbits with isotropy group $G(m, n)$ with $(m, n)=1$ and all other points to principal orbits. Then the map $\pi$ has a cross-section. Moreover any cross-section on $S^{1} \times 1$ may be extended to a cross-section over $X^{*}$.

The proof is analogous to that of (1.6) and we shall omit it. The total space is $X=D^{2} \times S^{1} \times S^{1}$ and the action is rotation of the first and second factors.

1.8. Lemma. Let $\pi: X \rightarrow X^{*}$ as above and assume that $X^{*}=[-1,1] \times I$, where $[-1,0) \times 0$ has stability group $G(m, n),(0,1] \times 0$ has stability group $G(p, q)$ such that $\left|\begin{array}{ll}p & m \\ a & n\end{array}\right|= \pm 1,0 \times 0$ has stability group $G$ and all other points correspond to principal orbits. Then there is a cross-section to this action. Moreover any cross-section on the $\operatorname{arc} A=(-1 \times I) \cup(1 \times I)$ may be extended to a cross-section over $X^{*}$.

Proof. Let $Y=X$-fixed point. The orbit space of this invariant locally compact subspace is $Y^{*}=X^{*}-(0 \times 0)$. A section over $Y^{*} \cap([-1,0] \times I)$ can be given according to (1.6); in fact, the section may be required to extend a given section on $A$. The restriction of this section to $[0,1] \times I$ may be extended to all of $[0,1] \times I$ $-(0 \times 0)$ by a slight modification of (1.6). This section extends trivially to all of $X^{*}$.

1.9. Remark. We could actually refine the above lemma to apply when a section is specified on all of $\partial X^{*}$ or on any finite set of disjoint intervals of $\partial X^{*}$.

Now assume that $\pi: X \rightarrow X^{*}$ is as above and $X^{*}$ is a compact 2-manifold with nonempty boundary. Let all interior points of $X^{*}$ correspond to principal orbits. On $\partial X^{*}$ we assume that there are two types of orbits only. A point on $\partial X^{*}$ may be the image of a $C$-orbit or it may be a fixed point. In the former case a component consists of either a whole circle or an open interval whose end points are fixed points. In the latter case we assume that a fixed point is isolated and the two open intervals with stability groups $G\left(m_{1}, n_{1}\right)$ and $G\left(m_{2}, n_{2}\right)$ that it separates have $\left|\begin{array}{ll}m_{1} & m_{2} \\ n_{1} & n_{2}\end{array}\right|= \pm 1$.

1.10. ThEOREM. $X$ is a closed orientable 4-manifold and the orbit map has a cross-section.

Proof. Consider the following possibilities:

Case 1. $X^{*}$ is a disc, $\partial X^{*}$ has no fixed points. Then (1.7) applies.

Case 2. $X^{*}$ is a disc and $\partial X^{*}$ has fixed points. Then we may assume that the number of fixed points is $t \geqq 2$ (by the condition $\left|\begin{array}{l}m_{i} \\ n_{i} \\ n_{i+1}\end{array} m_{i+1}\right|= \pm 1$ ). 


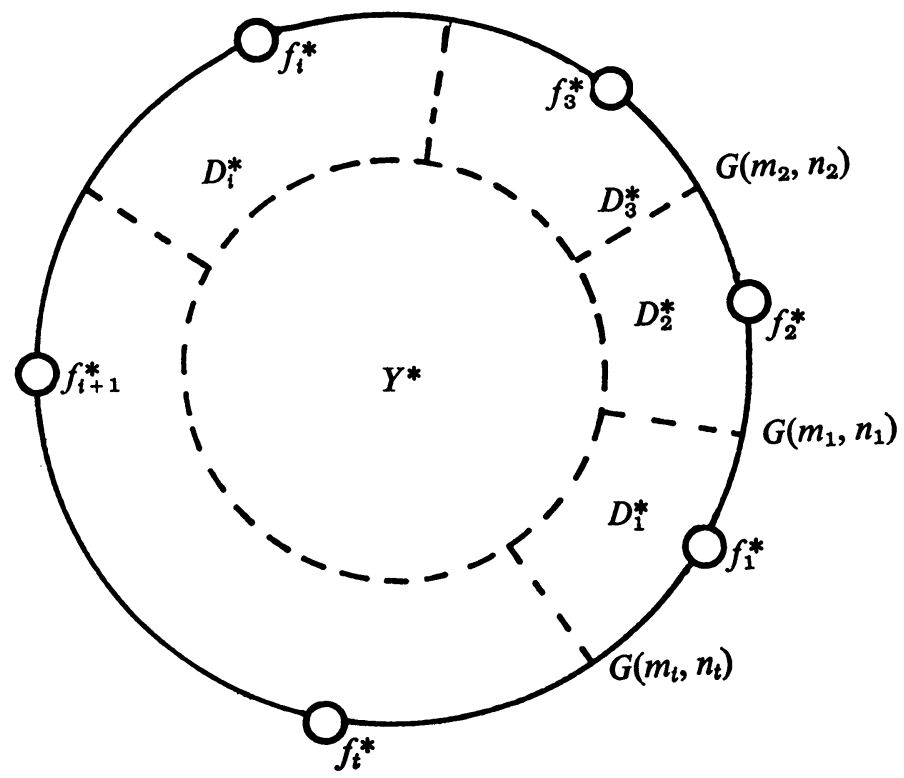

Thus $X^{*}$ looks as on the figure. We subdivide $X^{*}$ along the dotted lines. Each $D_{i}^{*}$ is just as in (1.8).

There is a section to the orbit map over $Y^{*}$ since $Y^{*}$ is a 2-cell. In fact more is true. We may choose a finite set of 2-cells $E_{1}^{*}, \ldots, E_{m}^{*}$ interior to $Y^{*}$ and specify cross-sections over them and extend to a cross-section over $Y^{*}$, since

$$
H^{2}\left(Y^{*}, E_{1}^{*} \cup \cdots \cup E_{m}^{*} ; Z \oplus Z\right)=0 .
$$

Similarly, we may remove the interiors of $E_{i}^{*}$ from $Y^{*}$ and specify cross-sections over $\partial E_{i}^{*}$. These can be extended to a cross-section over $Y^{*}-\left(E_{1}^{\circ *} \cup \cdots \cup E_{m}^{\circ *}\right)$ since

$$
H^{2}\left(Y^{*}-\left(E_{1}^{\circ *} \cup \cdots \cup E_{m}^{\circ *}\right), \partial E_{1}^{*} \cup \cdots \cup \partial E_{m}^{*} ; Z \oplus Z\right)=0 .
$$

The cross-section over $Y^{*}$ can be extended to $D_{1}^{*}$ by $(1.8)$, then $D_{2}^{*}, \ldots, D_{t}^{*}$ by (1.9).

Note that a by-product of this discussion is that we can construct a crosssection to the annular region $X^{*}-Y^{\circ *}$ extending any given cross-section on $\partial Y^{*}$.

Case 3. If $X^{*}$ is the general 2-manifold of the theorem, consider closed annular neighborhoods of the boundary components. Let their union be denoted $U^{*}$. The action over $Y^{*}=X^{*}-U^{\circ *}$ is trivial since $H^{2}\left(Y^{*} ; Z \oplus Z\right)=0$, thus we may choose a section over $Y^{*}$. We extend this section to all of $X^{*}$ either by Case 2 or (1.7).

The fact that $X$ is an orientable closed 4-manifold follows from (1.7) away from the fixed points. In a neighborhood of a fixed point we look at the inverse image of a small arc going from an orbit of type $G\left(m_{i-1}, n_{i-1}\right)$ to one of type $G\left(m_{i}, n_{i}\right)$ with 
interior orbits being principal orbits. The inverse image of this arc is an invariant 3 -sphere. This can be seen directly or the reader may consult $\$ 2$. The 2 -cell, in the orbit space, cut off by the arc has as inverse image the equivariant cone over this invariant 3-sphere.

1.11. Remark. It is not necessary to assume that $X$ and $X^{*}$ are compact. The above argument is valid if $X$ is a locally compact Hausdorff space and $X^{*}$ is either a 2-manifold with nonempty boundary or an open 2-manifold. If the orbit structure is as in (1.10) then the resulting space $X$ is a 4-manifold without boundary.

1.12. On the other hand, let us start with a $G$-action on a 4-manifold without boundary subject to the conditions:

(i) there are no nontrivial finite isotropy groups,

(ii) if all orbits are principal, then the bundle is trivial.

If $F \cup C=\varnothing$ then the above conditions imply that the manifold is a product.

If $F \cup C \neq \varnothing$ then we obtain the following result:

THEOREM. The orbit space is a 2-manifold with boundary, with "weights" on the boundary corresponding to the isotropy subgroups. The action has a cross-section. Therefore two such actions are equivalent if and only if there exists a "weight preserving" homeomorphism of the orbit spaces.

In the smooth case the theorem follows by utilizing (1.3) and (1.4) (compactness is not necessary). In the topological case and the general cohomological case the argument is completed in the appendix. The oriented, smooth and closed version of the theorem is stated in a more precise and numerical form in (4.4).

Finally we should point out to the reader that if the action of (1.12) is smooth, then the orbit map is smooth and we may choose a smooth cross-section and perform all of the above constructions of $\$(1.6)-(1.10)$, provided we smooth corners as needed.

2. Actions of $G$ on 3-manifolds. In this section we give an explicit description of the topological actions of $G$ on orientable 3-manifolds, $N$, with orbit space $N / G=N^{*}$.

By (1.1) there is a 2-dimensional orbit and it is easily seen that $N^{*}$ is a 1-manifold.

It is also easy to see that the only possible nontrivial stability group is a circle group and the image of an orbit with nontrivial stability group is a boundary point of $N^{*}$.

2.1. If $N^{*}$ is a circle, then all points of $N^{*}$ correspond to principal orbits. Thus $N$ is a $T^{2}$ bundle over $S^{1}$. Such principal bundles are classified up to bundle equivalence by

$$
\left[S^{1} ; K(Z, 2) \times K(Z, 2)\right]=H^{2}\left(S^{1} ; Z \oplus Z\right)=0 .
$$

Thus $N=N^{*} \times T^{2}=S^{1} \times T^{2}$ is a 3-torus and the action is the standard action.

2.2. $N^{*}$ is a closed interval with both boundary points corresponding to principal orbits. Clearly $N=N^{*} \times T^{2}$ with standard action and $N^{*}$ is a cross-section to the orbit map. 
2.3. $N^{*}$ is a closed interval with one boundary point corresponding to a principal orbit and the other has stability group $G(m, n)$. Since we have a cross-section $\chi: N^{*} \rightarrow N$, where $N$ is $I \times T^{2}$ with $0 \times T^{2}$ collapsed to the orbit space of the action of $G(m, n)$ on $0 \times T^{2}$. This is a solid torus, $N=D^{2} \times S^{1}$. The orientation is obtained from the orientation of $I$ and of $G=T^{2}$, collapsing each $0 \times S^{1} \times y$ to $0 \times 0 \times y$ for all $y \in S^{1}$. We parametrize $I \times S^{1} \times S^{1}$ by $(\rho, \alpha, \beta), 0 \leqq \rho \leqq 1,0 \leqq \alpha<2 \pi, 0 \leqq \beta<2 \pi$. The linear action

$$
\begin{aligned}
G \times N & \rightarrow N, \\
(\varphi, \theta) \times(\rho, \alpha, \beta) & \rightarrow(\rho, \alpha+p \varphi+q \theta, \beta+m \varphi+n \theta)
\end{aligned}
$$

is effective if and only if $\left|\begin{array}{ll}p & m \\ a & n\end{array}\right|= \pm 1$.

Since there is a cross-section to the orbit map, the action is either equivalent to this linear action or to the one reversing the orientation of $\chi\left(N^{*}\right)$ :

$$
(\varphi, \theta) \times(\rho, \alpha, \beta) \rightarrow(1-\rho, \alpha+p \varphi+q \theta, \beta+m \varphi+n \theta) .
$$

Now suppose we have another action on $N$

$$
(\rho, \alpha, \beta) \rightarrow(\rho, \alpha+\bar{p} \varphi+\bar{q} \theta, \beta+\bar{m} \varphi+\bar{n} \theta)
$$

and there is an equivariant homeomorphism $g: N \rightarrow N$ covering the identity map of the cross-section $g_{*}: \chi\left(N^{*}\right) \rightarrow \chi\left(N^{*}\right)$, then we can write

$$
g:(\rho, \alpha, \beta) \rightarrow(\rho, x \alpha+y \beta, w \alpha+z \beta) .
$$

It follows that $w=0$ and $(\bar{m}, \bar{n})=z(m, n), z= \pm 1$. Furthermore if we let

then

$$
\varepsilon=\left|\begin{array}{cc}
p & m \\
q & n
\end{array}\right|, \quad \bar{\varepsilon}=\left|\begin{array}{cc}
\bar{p} & \bar{m} \\
\bar{q} & \bar{n}
\end{array}\right|
$$

$$
x=\varepsilon\left|\begin{array}{ll}
\bar{p} & m \\
\bar{q} & n
\end{array}\right|=\varepsilon \bar{\varepsilon} z, \quad y=\varepsilon\left|\begin{array}{cc}
p & \bar{p} \\
q & \bar{q}
\end{array}\right|
$$

and $g$ is orientation preserving exactly when $\left|\begin{array}{ll}x & y \\ w & z\end{array}\right|=\varepsilon \vec{\varepsilon}=1$.

In particular $\{(-m,-n),(p, q)\}$ and $\{(m, n),(-p,-q)\}$ represent reversed orientation to $\{(m, n),(p, q)\}$, while $\{(-m,-n),(-p,-q)\}$ represents the same.

2.4. If $N^{*}$ is an open interval, then $N=N^{*} \times T^{2}$ with the standard action. If $N^{*}$ is a half-closed interval, then the discussions of (2.2) and (2.3) apply.

2.5. $N^{*}$ is a closed interval with both boundary points corresponding to nonprincipal orbits whose stability groups are $G(m, n)$ and $G\left(m^{\prime}, n^{\prime}\right)$.

Consider a point $Q$ on $N^{*}$

$$
(m, n) \stackrel{Q}{\longrightarrow}-\left(m^{\prime}, n^{\prime}\right)
$$

representing a principal orbit which separates $N$ into two solid tori, $V$ and $V^{\prime}$. Then $N$ is $S^{3}, S^{2} \times S^{1}$ or a lens space.

Choose a cross-section $\chi: N^{*} \rightarrow N$ to the orbit map. An orientation of $N$ determines in a natural way an orientation on $N^{*}$. The orientation induced by $\chi$ 
on $\chi\left(N^{*}\right)$ together with the orientation of the orbits determines the given orientation of $N$. There exist equivariant homeomorphisms $k$ and $k^{\prime}$ of $D^{2} \times S^{1}$ to $V$ and $V^{\prime}$,

$$
\begin{aligned}
& k:(\rho, \alpha, \beta) \rightarrow[\varepsilon(n \alpha+q \beta), \varepsilon(-m \alpha+p \beta)] \cdot \chi(\rho / 2), \\
& k^{\prime}:(\rho, \alpha, \beta) \rightarrow\left[\varepsilon^{\prime}\left(n^{\prime} \alpha-q^{\prime} \beta\right), \varepsilon^{\prime}\left(-m^{\prime} \alpha+p^{\prime} \beta\right)\right] \cdot \chi(1-\rho / 2)
\end{aligned}
$$

(where $\varepsilon=\left|\begin{array}{ll}p & m \\ q & n\end{array}\right|, \varepsilon^{\prime}=\left|\begin{array}{ll}p_{q^{\prime}}^{\prime} & m^{\prime} \\ n^{\prime}\end{array}\right|$ and - denotes the group operation in $N$ ) so that $k^{\prime} \mid \partial V^{\prime}$ and $k \mid \partial V$ are equivariantly homeomorphic (or diffeomorphic). Now $k$ is equivariant with respect to the linear action

$$
(\rho, \alpha, \beta) \rightarrow(\rho, \alpha+p \varphi+q \theta, \beta+m \varphi+n \theta)
$$

on $D^{2} \times S^{1}$ while $k^{\prime}$ is equivariant with respect to

on $D^{2} \times S^{1}$.

$$
(\rho, \alpha, \beta) \rightarrow\left(\rho, \alpha+p^{\prime} \varphi+q^{\prime} \theta, \beta+m^{\prime} \varphi+n^{\prime} \theta\right)
$$

The orientation of $N$ determines orientations of $D^{2} \times S^{1}$ by $k^{-1}$ and $k^{\prime-1}$. As in (2.3) the orientation of $D^{2} \times S^{1}$ determined by $k^{-1}$ is in turn determined by

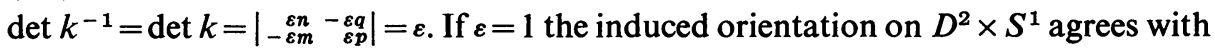
the standard one.

If $\varepsilon=-1$ the induced orientation on $D^{2} \times S^{1}$ is opposite to the standard one. In this case we precede $k$ by an orientation reversing homeomorphism

$$
g: D^{2} \times S^{1} \rightarrow D^{2} \times S^{1}, \quad g:(\rho, \alpha, \beta) \rightarrow(\rho,-\alpha, \beta)
$$

changing $\left\{\begin{array}{cc}p & m \\ q & n\end{array}\right\}$ into $\left\{\begin{array}{cc}\bar{D} & \bar{m} \\ \bar{q}\end{array}\right\}=\left\{\begin{array}{ll}-p & m \\ -q & n\end{array}\right\}$. The composition $k \circ g$ preserves the standard orientation of $D^{2} \times S^{1}$ and it is equivariant with respect to $\left\{\begin{array}{ll}\bar{D} & \bar{m} \\ \bar{q} & \bar{n}\end{array}\right\}$ where $\left|\begin{array}{ll}\bar{D} & \bar{m} \\ \bar{q} & \bar{n}\end{array}\right|=1$.

Thus we may assume that given the action of $G$ on the oriented $N$, there exists an orientation preserving equivariant homeomorphism of $D^{2} \times S^{1}=V$ into $N$ such that the standard action on $V$

has $\varepsilon=\left|\begin{array}{ll}p & m \\ q & n\end{array}\right|=1$.

$$
(\varphi, \theta) \times(\rho, \alpha, \beta) \rightarrow(\rho, \alpha+p \varphi+q \theta, \beta+m \varphi+n \theta)
$$

A similar argument shows that the orientation on the second solid torus, $V^{\prime}=D^{2} \times S^{1}$ is determined by the value $-\varepsilon^{\prime}$ and we may assume $\varepsilon^{\prime}=\left|\begin{array}{ll}p^{\prime} & m^{\prime} \\ q^{\prime}\end{array}\right|=-1$ to give the usual orientation on $V^{\prime}$.

The two solid tori $V$ and $V^{\prime}$ are sewn together equivariantly by an orientation reversing homeomorphism $h$ along their boundaries as follows:

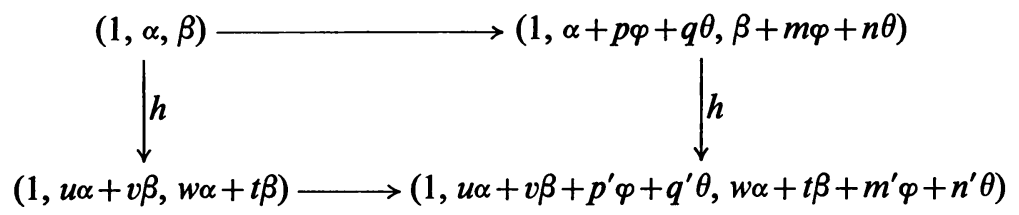

Therefore we must have

$$
\begin{aligned}
& u(\alpha+p \varphi+q \theta)+v(\beta+m \varphi+n \theta)=u \alpha+v \beta+p^{\prime} \varphi+q^{\prime} \theta \\
& w(\alpha+p \varphi+q \theta)+t(\beta+m \varphi+n \theta)=w \alpha+t \beta+m^{\prime} \varphi+n^{\prime} \theta
\end{aligned}
$$


Thus we obtain

$$
\begin{array}{ll}
u=\varepsilon\left|\begin{array}{ll}
p^{\prime} & m \\
q^{\prime} & n
\end{array}\right|, & v=\varepsilon\left|\begin{array}{ll}
p & p^{\prime} \\
q & q^{\prime}
\end{array}\right|, \\
w=\varepsilon\left|\begin{array}{ll}
m^{\prime} & m \\
n^{\prime} & n
\end{array}\right|, & t=\varepsilon\left|\begin{array}{ll}
p & m^{\prime} \\
q & n^{\prime}
\end{array}\right|,
\end{array}
$$

where $\varepsilon=\left|\begin{array}{ll}p & m \\ a & n\end{array}\right|$. Let $\varepsilon^{\prime}=\left|\begin{array}{ll}p^{\prime} & m^{\prime} \\ q^{\prime} & n^{\prime}\end{array}\right|$. Then we find that $\left|\begin{array}{cc}u & v \\ w & t\end{array}\right|=\varepsilon \varepsilon^{\prime}$.

From the above we may assume that $\varepsilon=1, \varepsilon^{\prime}=-1$ and therefore $h$ is in fact orientation reversing.

In order to decide what the manifold $N$ is, note that in $V^{\prime}$ the curve $\{(1, \alpha, \beta) \mid w \alpha+t \beta=0\}$ is null homotopic.

Thus when we sew $D^{2} \times S^{1}$ to $D^{2} \times S^{1}$ along their boundaries to form $N=V \cup_{h} V^{\prime}$, we obtain the oriented lens space $L(r, s)$, where

$$
r=\left|\begin{array}{cc}
m^{\prime} & m \\
n^{\prime} & n
\end{array}\right|, \quad s=\left|\begin{array}{cc}
p & m^{\prime} \\
q & n^{\prime}
\end{array}\right| .
$$

Next we shall drop the assumption $\varepsilon=\left|\begin{array}{ll}p & m \\ a\end{array}\right|=1$ but, of course, retain $\varepsilon \cdot \varepsilon^{\prime}=-1$. Recall that the lens space $L(r, s)$ satisfies the identities

$$
L(r, s)=L(-r,-s)=\bar{L}(-r, s)=\bar{L}(r,-s)=\bar{L}(r, r-s),
$$

where - means reversed orientation.

For fixed $(m, n),\left(m^{\prime}, n^{\prime}\right)$ the choice of $(-p,-q)$ instead of $(p, q)$ changes $\varepsilon$ to -1 , hence we need $\varepsilon^{\prime}=1$ and may choose $\left(-p^{\prime},-q^{\prime}\right)$. We see from the above that we obtain $L(w, t)=\bar{L}(r, s)$. But since in this case $k$ and $k^{\prime}$ were also orientation reversing we conclude that again $N=L(r, s)$, independently of the $\operatorname{sign}(p, q)$ resp. $(-p,-q)$.

Finally we want to show that $N$ is also independent of $(p, q),\left(p^{\prime}, q^{\prime}\right)$. This is already true of $r=\left|\begin{array}{ll}m^{\prime} & m \\ n^{\prime}\end{array}\right|$. Since $N$ is independent of $\varepsilon$, let us assume that $\varepsilon=1$. From $p n^{\prime}-q m^{\prime}=s, p n-q m=\varepsilon=1$, we obtain for $r \neq 0,1$,

$$
p=\left(m^{\prime}-s m\right) / r, \quad q=\left(n^{\prime}-s n\right) / r .
$$

Thus $m s \equiv m^{\prime} \bmod r$ and $n s \equiv n^{\prime} \bmod r$ determine $s$ uniquely in the interval $-|r|<s<|r|$. It is easy to show that the two congruences above determine the same $s$. They are in fact consequences of each other.

As a conclusion we have that the action, whose oriented orbit space is $(m, n)$ $\rightarrow-\left(m^{\prime}, n^{\prime}\right)$ and whose orbits are oriented by the action, takes place on the oriented lens space $L(r, s)$ with

$$
r=\left|\begin{array}{ll}
m^{\prime} & m \\
n^{\prime} & n
\end{array}\right| \text { and } \begin{array}{cc}
m s \equiv m^{\prime} & \bmod r \\
n s \equiv n^{\prime} & \bmod r .
\end{array}
$$

For $r=0$ we have $(m, n)= \pm\left(m^{\prime}, n^{\prime}\right)$ and $N=S^{2} \times S^{1}$. For $r=1, N=S^{3}$. We shall discuss these exceptional cases in (3.10).

2.6. Note that the orientation reversing map

$$
\rho \rightarrow 1-\rho
$$


on $\chi\left(N^{*}\right)$ results in an orbit space

$$
\left(m^{\prime}, n^{\prime}\right) \longrightarrow(m, n)
$$

and the total space $L(\bar{r}, \bar{s})$ has clearly $\bar{r}=-r$ and $s \bar{s} \equiv 1 \bmod r$.

Since $L(\bar{r}, \bar{s})=L(-r, \bar{s})=-L(r, \bar{s})$ we see that there is an orientation preserving homeomorphism between $L(r, s)$ and $L(r, \bar{s})$.

This is, of course, well known.

Actually, granting only that every homeomorphism of the torus is isotopic to $(\alpha, \beta) \rightarrow(u \alpha+v \beta, w \alpha+t \beta)$, where $\left|\begin{array}{ll}u & v \\ w & t\end{array}\right|= \pm 1$, and defining $L(r, s)$ as the sewing of two solid tori, the preceding arguments need only slight modifications to prove all the known homeomorphism relations almong lens spaces.

2.7. Finally consider $(m, n) \rightarrow\left(-m^{\prime},-n^{\prime}\right)$, that is, replace $\left(m^{\prime}, n^{\prime}\right)$ by $\left(-m^{\prime},-n^{\prime}\right)$, then

$$
L(\bar{r}, \bar{s})=L(-r,-s)=L(-r, r-s)
$$

since $-s \equiv r-s \bmod r$. Similarly replacing $(m, n)$ by $(-m,-n)$, gives

$$
L(\bar{r}, \bar{s})=L(-r,-s)=L(-r, r-s), \quad \bar{s}=\left|\begin{array}{cc}
-p & m^{\prime} \\
-q & n^{\prime}
\end{array}\right|=-s .
$$

Note that $L(r, s)=L(-r, r-s)$.

\section{Examples.}

3.1. Let $B$ be a closed 2-manifold and $M=B \times T^{2}$. The action of $G$ is trivial on $B$ and standard on $T^{2}$.

3.2. Let $N$ be a closed, orientable 3-manifold with an effective action of $S O(2)$. Let $M=N \times S^{1}$. Let $G$ act on $M$ by

$$
G \times N \times S^{1} \rightarrow N \times S^{1}, \quad(\varphi, \theta) \times(n, s) \rightarrow(\varphi(n), \theta(s)) .
$$

3.3. Let $N$ be a closed, orientable 3-manifold with an effective action of $G$. Let $M=N \times S^{1}$, where the action is trivial on the second factor.

3.4. Let $B$ be an orientable connected 2-manifold with nonempty boundary.

$$
\begin{aligned}
G \times B \times D^{2} \times S^{1} & \rightarrow B \times D^{2} \times S^{1}, \\
(\varphi, \theta) \times(b, \rho, \alpha, \beta) & \rightarrow(b, \rho, \alpha+p \varphi+q \theta, \beta+m \varphi+n \theta),
\end{aligned}
$$

where $\left|\begin{array}{ll}p & m \\ q & n\end{array}\right|= \pm 1$. The restriction of this action to the boundary, $M$, gives an effective action on a closed 4-manifold. The boundary of $B \times D^{2} \times S^{1}$ is $M=$ $\partial[(B \times I) \times I] \times S^{1}$, that is, $M=($ the double of $B \times I) \times S^{1}$. If $B$ is a surface of genus $g$ with $h$ discs removed, then the 4-manifold in question is

and

$$
M=\left[\left(S^{2} \times S^{1}\right) \# \cdots \#\left(S^{2} \times S^{1}\right)_{2 g+h-1}\right] \times S^{1} \text { if } g+h>1
$$

$$
M=S^{3} \times S^{1} \quad \text { if } g+h=1 .
$$

Another way to look at $M$ is as follows. The circle group $G(p, q)$ acts freely on $M$ and $N=M / G(p, q)$ is a closed 3-manifold with a semifree circle action. In fact, $G(m, n)$ acts freely on $N$ everywhere except for $h$ components of fixed circles 
corresponding to the boundary of $B$. Moreover $N / G(m, n)$ is $B$ with each boundary component of $B$ representing fixed points. By [8] we conclude that

and

$$
N=\left(S^{2} \times S^{1}\right)_{1} \# \cdots \#\left(S^{2} \times S^{1}\right)_{2 g+h-1} \text { if } g+h>1
$$

and clearly $M=N \times S^{1}$.

$$
N=S^{3} \quad \text { if } g+h=1,
$$

3.5. By virtue of the nature of the orbit structure and the cross-sectioning theorem (1.12) any action on a 4-manifold with all stability groups either the identity or $G(m, n)$ must be equivalent to that of (3.4). It follows from $\$ 2$ that the $N$ of (3.3) is a 3-dimensional torus or a lens space $L(r, s)$ and the orbit space is a torus or annulus whose boundary components are weighted by $G(m, n)$ and $G(p, q)$. Conversely, the cross-sectioning theorem tells us that an action on a closed oriented 4-manifold $M$ with orbit structure, a weighted annulus as just described, is equivalent to this type of action.

Thus, by just describing a specific action as in (3.3) and (3.4) (with favorable orbit types) we actually classify all possibilities with that given orbit structure. Furthermore, in the appendix, we shall show that the cross-sectioning theorem and the nature of the orbit structure remains valid in the continuous case. Consequently, this classification is also valid in the general continuous case.

3.6. Let us consider an orbit space homeomorphic to the 2-disk and with orbit structure as specified:

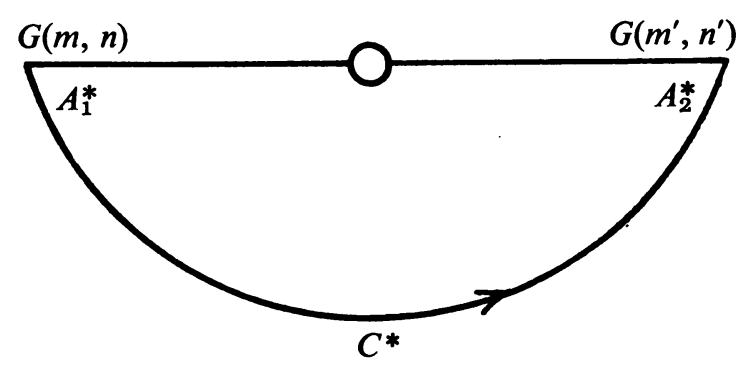

The straight arc $A_{1}^{*}$ has orbit type $G(m, n)$ except for its right end point. $A_{2}^{*}$ has orbit type $G\left(m^{\prime}, n^{\prime}\right)$ except for its left end point which corresponds to a fixed point. All other orbits are principal orbits. Ry a slight modification of (1.10) this action has a cross-section. Notice that the arc $C^{*}$ is the orbit space of an invariant lens space. Consequently, by (3.5), the original space $X$ is the cone over a lens space with the action extended to the cone from the boundary lens space in the standard manner. The space $X$ is a manifold with boundary (in fact a 4-cell), if and only if the lens space is a 3-sphere. This is equivalent to demanding $\left|n_{n^{\prime}}^{m^{\prime}}{ }_{n}^{m}\right|= \pm 1$. Since we shall not discuss anything but the case where $\left|\begin{array}{cc}m^{\prime} & m \\ n^{\prime}\end{array}\right|= \pm 1$ until the appendix, we shall tacitly assume that the determinant's value is \pm 1 .

3.7. Consider the linear action $G \times D^{2} \times D^{2} \rightarrow D^{2} \times D^{2}$ defined by

$$
(\varphi, \theta) \times\left(\rho_{1}, \alpha_{1}, \rho_{2}, \alpha_{2}\right) \rightarrow\left(\rho_{1}, \alpha_{1}+m^{\prime} \varphi+n^{\prime} \theta, \rho_{2}, \alpha_{2}+m \varphi+n \theta\right) .
$$


The action is effective if and only if $\left|\begin{array}{ll}m^{\prime} & m \\ n^{\prime}\end{array}\right|= \pm 1$. The orbit structure is as defined in (3.6) and consequently each topological action is equivalent to a linear action as described above. This action is clearly the extension to the cone of the linear action on the boundary sphere $\partial\left(D^{2} \times D^{2}\right)$. The boundary sphere corresponds to having at least one of the $\rho_{1}$ and $\rho_{2}$ equal to 1 . It will be crucial in the next section to describe the equivariant classification in terms of orientation preserving equivariant homeomorphisms. Referring back to (3.6), the arc $C^{*}$ is oriented from the left end point of $A_{1}^{*}$ to the right end point of $A_{2}^{*}$. The boundary 3-sphere $\partial\left(D^{2} \times D^{2}\right)$ $=D^{2} \times \partial D^{2} \cup \partial D^{2} \times D^{2}$ is represented by the lens space $L(r, s)$ where $r=\left|\begin{array}{ll}m^{\prime} & m \\ n^{\prime}\end{array}\right|$ $=\varepsilon= \pm 1$, and $s=0$. Thus, the boundary 3 -sphere has orientation $\varepsilon$ in accordance with the conventions of $\S 2$.

3.8. More generally, consider the following oriented orbit space, $W^{*}$.

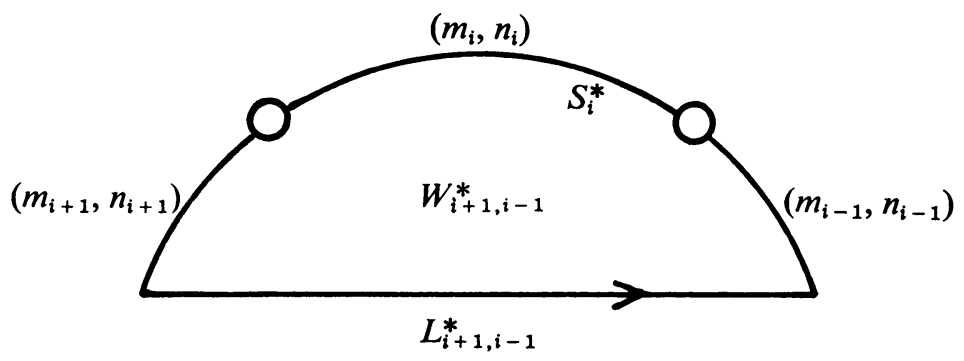

Let the interior points together with the interior points of the $\operatorname{arc} L^{*}$ correspond to principal orbits. The remainder of the boundary has two fixed points and three intervals with stability groups $G\left(m_{i-1}, n_{i-1}\right), G\left(m_{i}, n_{i}\right)$ and $G\left(m_{i+1}, n_{i+1}\right)$ where

$$
\left|\begin{array}{ll}
m_{i-1} & m_{i} \\
n_{i-1} & n_{i}
\end{array}\right|=\varepsilon_{i}= \pm 1 \text { and }\left|\begin{array}{cc}
m_{i} & m_{i+1} \\
n_{i} & n_{i+1}
\end{array}\right|=\varepsilon_{i+1}= \pm 1 \text {. }
$$

Note that the arc $S_{i}^{*}$ is the orbit space of an invariant 2-sphere $S_{i}$ in $W$.

From the proof of (1.10) it is clear that $W$ is an oriented 4-manifold with boundary the oriented lens space $L(r, s)$, where

$$
r=\left|\begin{array}{ll}
m_{i-1} & m_{i+1} \\
n_{i-1} & n_{i+1}
\end{array}\right|
$$

and $s m_{i+1} \equiv m_{i-1} \bmod r,-|r|<s<|r|$, if $r \neq 1$ or 0 .

Now, $m_{i-1} n_{i}-n_{i-1} m_{i}=\varepsilon_{i},-m_{i+1} n_{i}-n_{i+1} m_{i}=\varepsilon_{i+1}$; hence

Consequently,

$$
m_{i}=\frac{\left|\begin{array}{rr}
m_{i-1} & \varepsilon_{i} \\
-m_{i+1} & \varepsilon_{i+1}
\end{array}\right|}{\left|\begin{array}{rr}
m_{i-1} & -n_{i-1} \\
-m_{i+1} & n_{i+1}
\end{array}\right|}=\frac{\varepsilon_{i+1} m_{i-1}+\varepsilon_{i} m_{i+1}}{r}
$$

$$
\varepsilon_{i+1} m_{i-1}+\varepsilon_{i} m_{i+1} \equiv 0 \bmod r .
$$

Thus, $s=-\varepsilon_{i} \varepsilon_{i+1}, r \neq 0$, or 1 . This implies that

$$
L_{i+1, i-1}=L(r, s)=L\left(r,-\varepsilon_{i} \varepsilon_{i+1}\right)=-\varepsilon_{i} \varepsilon_{i+1} L(r, 1) .
$$


In other words, up to orientation, $L$ is a lens space of type $L(r, 1), r \neq 0,1$.

3.9. In fact, more is true. The manifold $W$ is an oriented 2-disk bundle over $S_{i}$. (In the topological case it is an associated topological $D^{2}$-bundle (not vectorbundle) associated to a principal $S^{1}$-bundle.) By definition, the characteristic class of the Hopf bundle is -1 . Hence, if the boundary of a $D^{2}$-bundle is $L(r, 1)$ then its characteristic class is $-r$.

The characteristic class of $W_{i+1, i-1}$.is therefore $\varepsilon_{i} \varepsilon_{i+1} r$ and hence the self intersection number of $S_{i}$ is

$$
\omega_{i}=S_{i} \cdot S_{i}=\varepsilon_{i} \varepsilon_{i+r} r=\left|\begin{array}{ll}
m_{i-1} & m_{i} \\
n_{i-1} & n_{i}
\end{array}\right|\left|\begin{array}{cc}
m_{i} & m_{i+1} \\
n_{i} & n_{i+1}
\end{array}\right|\left|\begin{array}{cc}
m_{i-1} & m_{i+1} \\
n_{i-1} & n_{i+1}
\end{array}\right| .
$$

It still remains to describe the self intersection number when $r=0$, and 1 . Of course if $r=0$, the self intersection number is 0 since the bundle is trivial. Note that the formula is still valid in this case, since $r=0$.

In order to cover the case $r=1$ we shall now give an alternative proof. The 2-disk bundle over the 2-sphere $S_{i}$ can be obtained by pasting together along the equator of $S_{i}$ two 2-disk bundles over the upper and lower hemispheres.

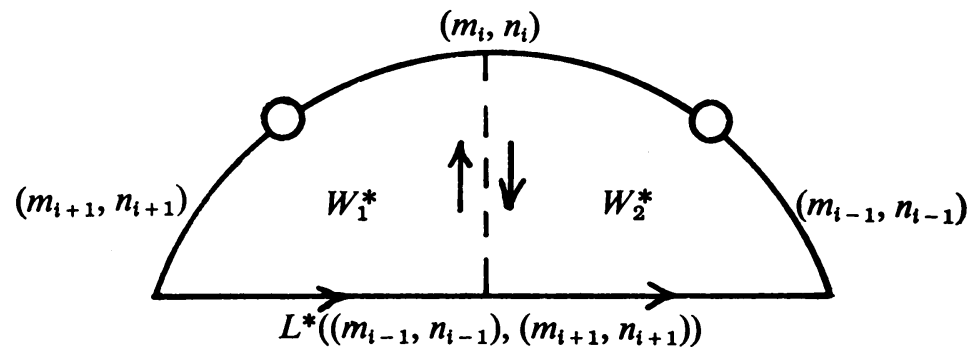

We want to show that $L\left(\left(m_{i-1}, n_{i-1}\right),\left(m_{i+1}, n_{i+1}\right)\right)=L(r, s)=-\varepsilon_{i} \varepsilon_{i+1} L(r, 1)$. We regard $L(r, s)$ as given to us as the lens space with oriented orbit space

$$
\left(m_{i+1}, n_{i+1}\right) \longrightarrow\left(m_{i-1}, n_{i-1}\right) \text {. }
$$

Following (2.5) we split $L(r, s)$ up into the union of two solid tori. We choose, in the terminology of $(2.5),(m, n)=\left(m_{i+1}, n_{i+1}\right),\left(m^{\prime}, n^{\prime}\right)=\left(m_{i-1}, n_{i-1}\right)$ and $(p, q)$ $=\left(m_{i}, n_{i}\right)$. If

then

$$
\varepsilon=\left|\begin{array}{cc}
p & m \\
q & n
\end{array}\right|=\left|\begin{array}{cc}
m_{i} & m_{i+1} \\
n_{i} & n_{i+1}
\end{array}\right|=\varepsilon_{i+1}=1,
$$

Thus,

$$
\begin{aligned}
& r=\left|\begin{array}{ll}
m^{\prime} & m \\
n^{\prime} & n
\end{array}\right|=\left|\begin{array}{ll}
m_{i-1} & m_{i+1} \\
n_{i-1} & n_{i+1}
\end{array}\right|, \\
& s=\left|\begin{array}{ll}
p & m^{\prime} \\
q & n^{\prime}
\end{array}\right|=\left|\begin{array}{ll}
m_{i} & m_{i-1} \\
n_{i} & n_{i-1}
\end{array}\right|=-\varepsilon_{i} .
\end{aligned}
$$

$$
\begin{aligned}
L(r, s) & =L\left(\left|\begin{array}{ll}
m_{i-1} & m_{i+1} \\
n_{i-1} & n_{i+1}
\end{array}\right|,-\left|\begin{array}{ll}
m_{i-1} & m_{i} \\
n_{i-1} & n_{i}
\end{array}\right|\right) \\
& =-\varepsilon_{i}(1) L(r, 1)=-\varepsilon_{i} \varepsilon_{i+1} L(r, 1) .
\end{aligned}
$$


On the other hand, if $\varepsilon=-1=\varepsilon_{i+1}$, then

$$
t=\left|\begin{array}{ll}
-p & m^{\prime} \\
-q & n^{\prime}
\end{array}\right|=\left|\begin{array}{ll}
m_{i} & m_{i-1} \\
n_{i} & n_{i-1}
\end{array}\right|=\left|\begin{array}{ll}
m_{i-1} & m_{i} \\
n_{i-1} & n_{i}
\end{array}\right|=-\varepsilon_{i} .
$$

Hence $L(w, t)$ (in the notation of $(2.5)$ ) is

$$
L\left(\left|\begin{array}{ll}
m_{i-1} & m_{i+1} \\
n_{i-1} & n_{i+1}
\end{array}\right|,-\varepsilon_{i}\right)=L\left(r,-\varepsilon_{i}\right)=-\varepsilon_{i} L(r, 1)=-L(r, s) .
$$

Hence $L(r, s)=\varepsilon_{i} L(r, 1)$. Since $\varepsilon_{i+1}=-1$, in the last case, $L(r, s)$ is once again $-\varepsilon_{i+1} \varepsilon_{i} L(r, 1)$. We have therefore shown that the 2-sphere $S_{i}$, over which $W$ is an oriented 2-disk bundle, has self intersection number $\varepsilon_{i+1} \varepsilon_{i} r$, for every value of $r$.

4. Equivariant classification. In this section we shall give an equivariant classification for actions without finite stability groups $(E=\varnothing)$ and where either $F \cup C \neq \varnothing$ or the manifold is not compact. This section may be viewed as a numerical form of (1.10) and (1.12).

4.1. Weighted orbit spaces and orientation. Let $G$ act smoothly on a 4-manifold $M$ with orbit space $M^{*}$. We shall now make precise the remarks in (1.12). Each orbit in $M^{*}$ has associated to it a certain orbit type which is characterized by the stability group of the points on the orbit together with the slice representation at the given orbit. The slice representation at a fixed point is just the action of the stability group on the normal disk. This action is determined in the case of a fixed point $f_{i}^{*}$ by the pairs $\pm G\left(m_{i}, n_{i}\right)$, that is $G\left(m_{i}, n_{i}\right)$ or $G\left(-m_{i},-n_{i}\right)$, and

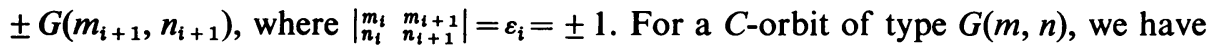
the standard representation. For an $E$-orbit of type $Z_{q} \subset G$, we have the particular linear representation of this finite cyclic group on the rotations of the disk. This representation is described in terms of a pair of relatively prime integers. The orbit space together with its orbit types and slice representations is called a weighted orbit space.

Let $M^{*}$ and $M^{\prime *}$ denote the orbit spaces of a smooth action of $G$ on the closed oriented smooth 4-manifolds $M$ and $M^{\prime}$. A diffeomorphism (homeomorphism) of $M^{*}$ and $M^{\prime *}$ which carries the weights of $M^{*}$ isomorphically onto the weights of $M^{\prime *}$ is called a weight preserving diffeomorphism (homeomorphism).

For classification purposes it is often convenient to work with a specific orientation. We orient the group $G$ once and for all and then an orientation of $M$ determines an orientation of $M^{*}$ and vice versa, provided that there are no stability groups of the type which reverse the orientation of a slice. The orientation of $M$ can be described as the product orientation of the set of principal orbits-this bundle is an oriented bundle. When the orbit map has a cross-section then we always assume that the orientation of the image of $M^{*}$, via the cross-section, is that induced by the cross-section and that the orientation of $M$ is consequently compatible with it. In particular, suppose we have a portion $D^{*}$ of the orbit space $M^{*}$ : 


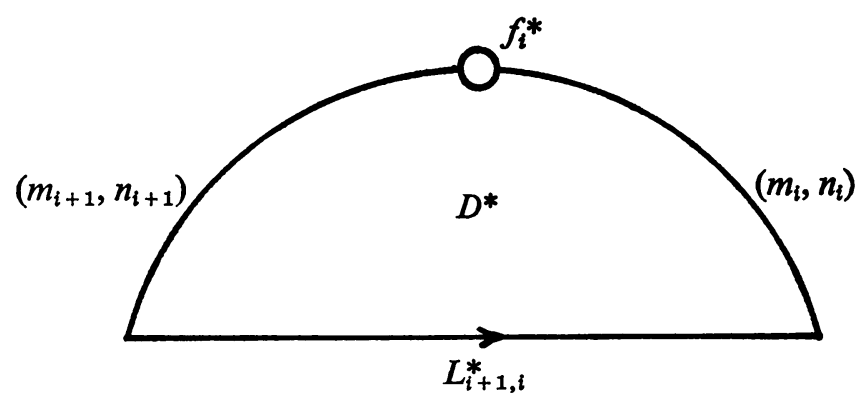

$D$ is a 4-disk and if the orbit space is assumed to be oriented as indicated the 4-disk becomes a cone over the 3-sphere $L_{i+1, i}$ with orientation $\left|\begin{array}{cc}m_{i} & m_{i+1} \\ n_{i+1}\end{array}\right|=\varepsilon_{i}$ times the standard oriented 4-disk. (Here the cross-section lifts the oriented 2-disk $D^{*}$ into the 4-disk $D$ and the product orientation of $D^{*} \times G$ induces the orientation on the 4-disk via the map $D^{*} \times G \rightarrow G\left(\chi\left(D^{*}\right)\right)=D$, where $X$ denotes the cross-section.)

4.2. Let $G$ act on the 4-manifolds $M$ and $M^{\prime}$ such that the orbit maps have cross-sections $\chi$ and $\chi^{\prime}$.

From $\S 1$ it follows that if $G$ acts smoothly and effectively on an oriented closed smooth 4-manifold, then the orbit map has a cross-section if and only if there are no finite stability groups and if all orbits are principal then the principal bundle is trivial.

THEOREM. Let $G$ act effectively and smoothly on the closed smooth orientable 4-manifolds $M$ and $M^{\prime}$ such that the orbit map has a cross-section. Then there exists an equivariant diffeomorphism $h$ of $M$ onto $M^{\prime}$ if and only if there exists a weight preserving diffeomorphism $h^{*}$ of $M^{*}$ onto $M^{\prime *}$. Furthermore, if $M$ and $M^{\prime}$ are oriented and the orientation of $M^{*}$ and $M^{\prime *}$ are those induced by $M$ and $M^{\prime}$, then $h$ is orientation preserving if and only if $h^{*}$ is orientation preserving.

4.3. Of particular value for the topological classification of $M$, i.e. the identification of $M$ in terms of its weighted orbit space, is the case when $M^{*}$ is an oriented 2-disk.

If $M$ has no fixed points then $M=S^{3} \times S^{1}$ by (3.4). Otherwise we assert that $M$ is simply connected.

To see this fact remove a small equivariant 4-cell about a fixed point. This corresponds, using the cross-section, to removing a small closed 2-cell about the image of the fixed point in the orbit space. The cross-section now retracts to the remaining boundary. This deformation lifts equivariantly to a deformation of the manifold to a linear chain of 2-spheres. Their number equals the number of fixed points minus two. The fundamental group of the total space is therefore trivial by Van Kampen's theorem.

Let us now represent this 4-manifold in terms of its weighted orbit space. By

$$
M=\left\{\left(m_{1}, n_{1}\right),\left(m_{2}, n_{2}\right), \ldots,\left(m_{t}, n_{t}\right)\right\}
$$


we mean the simply connected oriented 4-manifold whose weighted orbit space is as described in (4.1). We may assume without loss of generality that $M^{*}$ is oriented from $\left(m_{i}, n_{i}\right)$ to $\left(m_{i+1}, n_{i+1}\right)$.

Suppose $M$ and $M^{\prime}$ are simply connected oriented 4-manifolds described as above. Then we obtain the following result.

THEOREM. There is an orientation preserving equivariant diffeomorphism between $M=\left\{\left(m_{1}, n_{1}\right),\left(m_{2}, n_{2}\right), \ldots,\left(m_{t}, n_{t}\right)\right\}$ and $M^{\prime}=\left\{\left(m_{1}^{\prime}, n_{1}^{\prime}\right),\left(m_{2}^{\prime}, n_{2}^{\prime}\right), \ldots,\left(m_{t^{\prime}}^{\prime}, n_{t^{\prime}}^{\prime}\right)\right\}$ if and only if $t=t^{\prime}$ and for some fixed $k$ either $\left(m_{i}, n_{i}\right)=\left(m_{k+i}^{\prime}, n_{k+i}^{\prime}\right)$ or $\left(m_{i}, n_{i}\right)=$ $\left(-m_{k+i}^{\prime},-n_{k+i}^{\prime}\right)$, for $i=1, \ldots, t$.

4.4. Now we shall discuss the general case. Assume therefore that the action on $M$ has the following properties:

(i) There are no nontrivial finite isotropy groups.

(ii) If all orbits are principal, then the bundle is trivial.

These conditions imply that if $F \cup C=\varnothing$ then the manifold is a product. If $F \cup C \neq \varnothing$ then according to (1.12) $M$ has a cross-section and $M^{*}$ is a 2-manifold with boundary. Interior points correspond to principal orbits. Boundary points correspond to $C$-orbits or (isolated) fixed points. The weighted oriented orbit space completely determines the action up to orientation preserving equivariant diffeomorphism. It has the following arithmetic invariants:

$g \geqq 0$; the genus of the oriented 2-manifold $M^{*}$.

$s \geqq 0$; the number of boundary components of $M^{*}$ all of whose orbits are $C$-orbits.

$t \geqq 0$; the number of boundary components of $M^{*}$ each having fixed points.

$\varepsilon$; a specific orientation assigned to the orientable 2 -manifold $M^{*}$. Since an orientation for $G$ has been chosen once and for all, the orientation $\varepsilon$ determines an orientation for $M$.

$\left\langle p_{i}, q_{i}\right\rangle$; the $i$ th boundary component of $M^{*}$, consisting entirely of $C$-orbits with stability group $G\left(p_{i}, q_{i}\right)$ or equivalently $G\left(-p_{i},-q_{i}\right) .\left\langle p_{i}, q_{i}\right\rangle$ denotes either parametrization together with an orientation of the boundary circle compatible with $\varepsilon$.

$\{m, n\}_{i}$; the $i$ th boundary component of $M^{*}$, which contains $t_{i}$ fixed points, $t_{i} \geqq 2$, can be represented by $\{m, n\}_{i}=\left\{\left\langle m_{i, 1}, n_{i, 1}\right\rangle, \ldots,\left\langle m_{i, t_{i}}, n_{i, t_{i}}\right\rangle\right\}$. Here $\left\langle m_{i, j}, n_{i, j}\right\rangle$ $= \pm\left(m_{i, j}, n_{i, j}\right)$ is a parametrization of the stability group of the $j$ th oriented arc of the $i$ th boundary component. The order of the entries in $\{m, n\}$ is determined up to cyclic permutation.

With these numerical invariants one can translate the theorem of (1.12) to read:

Theorem. Suppose that $M$ and $M^{\prime}$ are closed, oriented 4-manifolds with Gactions such that $C \cup F \neq \varnothing$ and $E=\varnothing$. Then there is an equitariant orientation preserving diffeomorphism between $M$ and $M^{\prime}$ if and only if

$$
\begin{aligned}
\left\{\varepsilon ; g ; s ; t ;\left\langle p_{1}, q_{1}\right\rangle, \ldots,\left\langle p_{s}, q_{s}\right\rangle ;\{m, n\}_{1}, \ldots,\{m, n\}_{t}\right\} \\
=\left\{\varepsilon^{\prime} ; g^{\prime} ; s^{\prime} ; t^{\prime} ;\left\langle p_{1}^{\prime}, q_{1}^{\prime}\right\rangle, \ldots,\left\langle p_{s^{\prime}}^{\prime}, q_{s^{\prime}}^{\prime}\right\rangle ;\left\{m^{\prime}, n^{\prime}\right\}_{1}, \ldots,\{m, n\}_{t^{\prime}}\right\},
\end{aligned}
$$


where equality means identical except that $\langle p, q\rangle$ 's may be permuted and the $\{m, n\}$ 's may be permuted.

5. Topological classification of the simply connected $G$-manifolds. In this section, the smooth actions of the torus are classified on closed, smooth simply connected 4-manifolds. Actually the vanishing of $H_{1}(M ; Z)$ will imply simple connectedness. In the appendix we drop the assumption of differentiability without any weakening of the results. Furthermore, when we also drop the assumption of local Euclideaness the analogues of the results of this section will still be valid but the arguments of course are considerably more delicate.

We shall assume that $M$ is a smooth closed 4-manifold, $H_{1}(M ; Z)=0$ and $G$ acts smoothly and effectively on $M$.

\subsection{Lemma. The action has fixed points.}

Proof. Clearly, $H_{1}(M)=H^{3}(M)=0$, hence $H_{2}(M ; Z)$ is free and $H_{3}(M ; Z)=0$. The Euler characteristic of $M, \chi(M)=2+\operatorname{rank} H_{2}(M)$. It is well known that for a torus $G$, the Euler characteristic of the fixed point set $\chi(F ; G)=\chi(M)$. Thus $G$ acts with at least 2 fixed points.

Since $\pi_{1}(M) \rightarrow \pi_{1}\left(M^{*}\right)$ is surjective and the Hurewicz homomorphism is natural, the natural homomorphism $H_{1}(M ; Z) \rightarrow H_{1}\left(M^{*} ; Z\right)$ is surjective. $M^{*}$ is a 2 manifold, with the fixed points on the boundary, and hence $M^{*}$ is a 2-disk.

Let $f_{1}, f_{2}, \ldots, f_{t}$ denote the fixed points, $t \geqq 2$, and $f_{i}^{*}$ their images on $M^{*}$. The arc, $S_{i}^{*}$, between $f_{i}^{*}$ and $f_{i+1}^{*}$ on $\partial\left(M^{*}\right)$ represents an invariant 2-sphere $S_{i}$. Denote its stability group by $G\left(m_{i}, n_{i}\right)$.

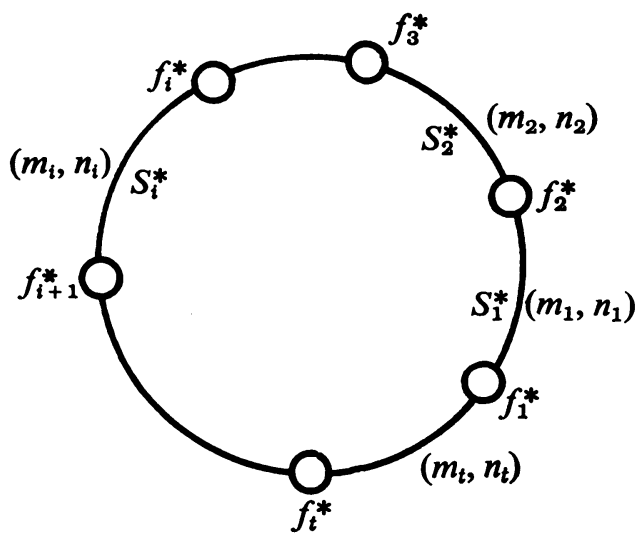

Recall that

$$
\begin{aligned}
\left|\begin{array}{ll}
m_{i-1} & m_{i} \\
n_{i-1} & n_{i}
\end{array}\right| & =\varepsilon_{i}= \pm 1, \quad i=2,3, \ldots, t \\
\left|\begin{array}{ll}
m_{t} & m_{1} \\
n_{t} & n_{1}
\end{array}\right| & =\varepsilon_{1}= \pm 1,
\end{aligned}
$$


where $\varepsilon_{i}$ is the orientation of $S^{3}$ viewed as the boundary of a neighborhood of the fixed point $f_{i}$ (see 3.7).

Next we show that all the interior points of the disk represent principal orbits.

5.2. LEMMA. Let $M$ be a closed, smooth orientable 4-manifold with an effective $G$ action. If $H_{1}(M ; Z)=0$, then the only finite isotropy group is the identity.

Proof. The homology argument above assures the existence of fixed points. By (1.4) if $y \in M, G_{y}=Z_{q} \subset G$, then $y^{*}$ is an isolated interior point of $M^{*}$ if $G_{y} \neq e$.

Let $A^{*}$ be an arc from some fixed point $f_{i}^{*}$ to $y^{*}$ in $M^{*}$ such that the interior points of $A^{*}$ correspond to principal orbits. Let $A$ be the lifting of $A^{*}$ to $M$. The saturation $X$ of $A$, i.e. $G(A)$, is the cone over a torus with the base of the cone collapsed by the free action of $Z_{q} \subset G$ on the toral base. Consequently, $H^{3}(X ; Z) \approx Z_{q}$. Therefore the exactness of

$$
0=H^{3}(M ; Z) \rightarrow H^{3}(X ; Z) \rightarrow H^{4}(M, X ; Z)
$$

and the freeness of $H^{4}(M, X ; Z)$ implies that $Z_{q}$ must be the identity.

5.3. Now we turn to the homeomorphism classification of these manifolds. Our building blocks are the manifolds with $2 \leqq t \leqq 4$.

Let in general $\bar{M}$ denote $M$ with reversed orientation and let $P=C P^{2}, Q_{0}=S^{2} \times S^{2}$ and $Q_{1}=P \# \bar{P}$, the other $S^{2}$ bundle over $S^{2}$. (The structure group in the smooth case is $S O(3)$. In the topological case, the group of orientation preserving homeomorphisms of $S^{2}$ onto itself deforms onto the rotation group $S O(3)$.)

5.4. $t=2$.

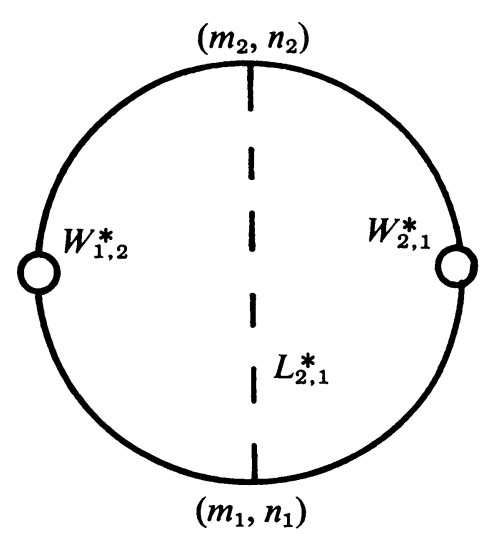

Since $\left|\begin{array}{ll}m_{1} & m_{2} \\ n_{1} & n_{2}\end{array}\right|=\varepsilon_{2}= \pm 1$ we have that $L_{2,1}=S^{3}$ and $M=W_{2,1} \cup W_{1,2}$. Both $W_{1,2}$ and $W_{2,1}$ are 4-cells, hence $M=S^{4}$ (see (3.6)). Thus there are infinitely many distinct actions corresponding to pairs $\left(m_{i}, n_{i}\right), i=1,2$. Notice that all possible distinct inequivalent actions are related to the action $\{(0,1),(1,0)\}$ by an automorphism of $G$, that is, if $\left|\begin{array}{cc}m_{1} & m_{2} \\ n_{1} & n_{2}\end{array}\right|=\varepsilon_{2}= \pm 1$, there exists an automorphism of $G$ sending $G(0,1)$ and $G(1,0)$ onto $G\left(m_{1}, n_{1}\right)$ and $G\left(m_{2}, n_{2}\right)$. 
5.5. $t=3$.

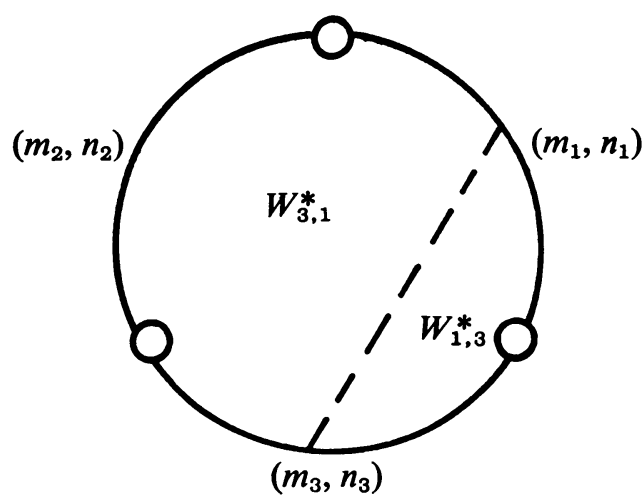

Note that $L_{3,1}=S^{3}$. Also $W_{1,3}$ is a 4-cell and $W_{3,1}$ is a $D^{2}$-bundle over $S_{2}$, with Euler class $\varepsilon_{2} \varepsilon_{3}\left|\begin{array}{ll}m_{1} & m_{3} \\ n_{3}\end{array}\right|=-\varepsilon_{1} \varepsilon_{2} \varepsilon_{3}=S_{2} \cdot S_{2}$. If $S_{2} \cdot S_{2}=1$ we have the complex projective plane $M=P$; if $S_{2} \cdot S_{2}=-1$, then $M=\bar{P}$.

Notice that, if $S_{2} \cdot S_{2}=1$ one can solve for $\left(m_{3}, n_{3}\right)$ :

$$
m_{3}=-\varepsilon_{1} \varepsilon_{2}\left(m_{2}+\varepsilon_{2} m_{1}\right), \quad n_{3}=\varepsilon_{1}\left(n_{1}+n_{2}\right) .
$$

It is easy to see that each action on $P$ arises from an action on the 4-disk. We have the standard action

$$
\left(\rho_{1}, \alpha_{1}, \rho_{2}, \alpha_{2}\right) \rightarrow\left(\rho_{1}, \alpha_{1}+m_{1} \varphi+n_{1} \theta, \alpha_{2}+m_{2} \varphi+n_{2} \theta\right)
$$

on the 4-disk. On the boundary 3-sphere $L_{2,1}$ the group $G\left(m_{3}, n_{3}\right)$ operates freely, it is in fact the Hopf fibering (or its inverse). If we collapse the boundary by the action of $G\left(m_{3}, n_{3}\right)$ we obtain $P$ (or $\left.\bar{P}\right)$ with a torus action as above. Obviously, there are an infinite number of inequivalent actions but once again any two are related by an automorphism of $G$.

5.6. $t=4$.

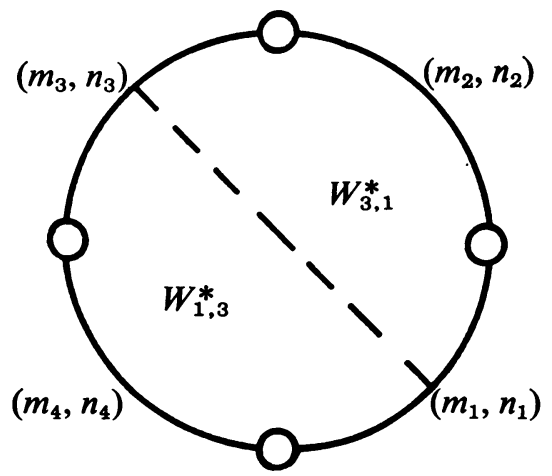

Let $r_{i}=\left|\begin{array}{cc}m_{i}-1 & m_{i+1} \\ n_{i}-1 & n_{i+1}\end{array}\right|$, counting mod 4. In particular,

$$
r_{2}=\left|\begin{array}{ll}
m_{1} & m_{3} \\
n_{1} & n_{3}
\end{array}\right|, \quad r_{3}=\left|\begin{array}{ll}
m_{2} & m_{4} \\
n_{2} & n_{4}
\end{array}\right| .
$$


Observe that $M$ is the union of two $D^{2}$-bundles, $W_{3,1}$ with 0 -section $S_{2}$ and $W_{1,3}$ with 0 -section $S_{4}$. They are pasted together along their boundaries $L_{3,1}$ and $L_{1,3}$. Furthermore, $M$ may also be constructed from the $W_{4,1}$ and $W_{1,4}$ over $S_{3}$ and $S_{1}$. If $r_{2}=0$, then $G\left(m_{1}, n_{1}\right)= \pm G\left(m_{3}, n_{3}\right)$ and $L_{3,1}$ would be $S^{2} \times S^{1}$. When $r_{2}=0$, the structure groups $G\left(m_{1}, n_{1}\right)$ and $G\left(m_{3}, n_{3}\right)$ are equal and hence one can easily see that $W_{4,2}$ and $W_{2,4}$ are pasted together to form an $S^{2}$-bundle over $S_{3}$ (or $S_{1}$ ). Thus $M$ is either $Q_{0}$ or $Q_{1}$ since the structure group $G\left(m_{1}, n_{1}\right)$ can be reduced to $S O(3)$.

We shall show that if both $r_{3}$ and $r_{2} \neq 0$, then necessarily $r_{2}$ or $r_{3}$ equals \pm 1 . Consequently, $M$ will be the union along a 3 -sphere of $D^{2}$-bundles with characteristic class \pm 1 . Hence, $M$ will be the connected sum $P$ with itself or $\bar{P}$ with itself. Consideration of the intersection matrix for $\mathrm{H}_{2}(M ; Z)$ will resolve the ambiguities according to the homotopy type classification of simply connected 4-manifolds [3].

First, if $r_{2}=0$, then $\left(m_{1}, n_{1}\right)= \pm\left(m_{3}, n_{3}\right)$. The intersection matrix for the generators $S_{2}$ and $S_{3}$ of $H_{2}(M ; Z)$ is

$$
\Lambda=\left(\begin{array}{cc}
0 & \varepsilon_{3} \\
\varepsilon_{3} & \varepsilon_{3} \varepsilon_{4} r_{3}
\end{array}\right) \sim\left(\begin{array}{cc}
0 & 1 \\
1 & \varepsilon_{3} \varepsilon_{4} r_{3}
\end{array}\right) .
$$

If $r_{3}$ is even, $M=Q_{0}=S^{2} \times S^{2}$, and if $r_{3}$ is odd, $M=Q_{1}=P \# \bar{P}$.

Suppose $r_{2} \neq 0$, then

$$
\left|\begin{array}{ll}
m_{1} & m_{2} \\
n_{1} & n_{2}
\end{array}\right|=\varepsilon_{2}, \quad\left|\begin{array}{ll}
m_{2} & m_{3} \\
n_{2} & n_{3}
\end{array}\right|=\varepsilon_{3}
$$

yield

$$
m_{2}=\left(\varepsilon_{2} m_{3}+\varepsilon_{3} m_{1}\right) / r_{2}, \quad n_{2}=\left(\varepsilon_{3} n_{1}+\varepsilon_{2} n_{3}\right) / r_{2} .
$$

On the other hand,

$$
\left|\begin{array}{ll}
m_{3} & m_{4} \\
n_{3} & n_{4}
\end{array}\right|=\varepsilon_{4}, \quad\left|\begin{array}{ll}
m_{4} & m_{1} \\
n_{4} & n_{1}
\end{array}\right|=\varepsilon_{1}
$$

yield

$$
m_{4}=\left(\varepsilon_{4} m_{1}+\varepsilon_{1} m_{3}\right) /-r_{2}, \quad n_{4}=\left(\varepsilon_{1} n_{3}+\varepsilon_{4} n_{1}\right) /-r_{2} .
$$

Thus we have

$$
\begin{array}{lll}
m_{3} \equiv-\varepsilon_{2} \varepsilon_{3} m_{1} & \bmod r_{2}, & n_{3} \equiv-\varepsilon_{2} \varepsilon_{3} n_{1}, \\
m_{3} \equiv-\varepsilon_{1} \varepsilon_{4} m_{1} & \bmod r_{2}, & n_{3} \equiv-\varepsilon_{1} \varepsilon_{4} n_{1}
\end{array}
$$

and

$$
\begin{array}{ll}
m_{2}=\left(\varepsilon_{2} / r_{2}\right)\left(m_{3}+\varepsilon_{2} \varepsilon_{3} m_{1}\right), & n_{2}=\left(\varepsilon_{2} / r_{2}\right)\left(n_{3}+\varepsilon_{2} \varepsilon_{3} n_{1}\right), \\
m_{4}=\left(-\varepsilon_{1} / r_{2}\right)\left(m_{3}+\varepsilon_{1} \varepsilon_{4} m_{1}\right), & n_{4}=\left(-\varepsilon_{1} / r_{2}\right)\left(n_{3}+\varepsilon_{1} \varepsilon_{4} n_{1}\right) .
\end{array}
$$

If $\varepsilon_{1} \varepsilon_{4}=-\varepsilon_{2} \varepsilon_{3}$, then we have

$$
2 m_{3}=r_{2}\left(\varepsilon_{2} m_{2}-\varepsilon_{1} m_{4}\right), \quad 2 n_{3}=r_{2}\left(\varepsilon_{2} n_{2}-\varepsilon_{1} n_{4}\right) .
$$

For $\left|r_{2}\right|>2$, we have a contradiction to the fact that $\left(m_{3}, n_{3}\right)$ are relatively prime. 
If $r_{2}=2 \delta, \delta= \pm 1$, then

$$
\begin{aligned}
& \left|\begin{array}{ll}
m_{2} & \delta\left(\varepsilon_{2} m_{2}-\varepsilon_{1} m_{4}\right) \\
n_{2} & \delta\left(\varepsilon_{2} n_{2}-\varepsilon_{1} n_{4}\right)
\end{array}\right|=\varepsilon_{3} \\
& \left|\begin{array}{ll}
\delta\left(\varepsilon_{2} m_{2}-\varepsilon_{1} m_{4}\right) & m_{4} \\
\delta\left(\varepsilon_{2} n_{2}-\varepsilon_{1} n_{1}\right) & n_{4}
\end{array}\right|=\varepsilon_{4}
\end{aligned}
$$

Thus, $m_{3}=\delta\left(\varepsilon_{2} m_{2}-\varepsilon_{1} m_{4}\right), n_{3}=\delta\left(\varepsilon_{2} n_{2}-\varepsilon_{1} n_{4}\right)$. Since $r_{3}=\delta \varepsilon_{2} \varepsilon_{4}= \pm 1$, we have $L_{4,2}=-\varepsilon_{3} \varepsilon_{4} L\left(r_{3}, 1\right)=-\varepsilon_{2} \varepsilon_{3} \delta S^{3}$, and consequently, $M=W_{2,4} \# W_{4,2}$.

The self intersection matrix for generators $S_{1}$ and $S_{3}$ is

$$
\Lambda=\left(\begin{array}{cc}
-\varepsilon_{1} \varepsilon_{4} \delta & 0 \\
0 & \varepsilon_{2} \varepsilon_{3} \delta
\end{array}\right)=\left(\begin{array}{cc}
\varepsilon_{2} \varepsilon_{3} \delta & 0 \\
0 & \varepsilon_{2} \varepsilon_{3} \delta
\end{array}\right)
$$

Hence, $M=P \# P$ or $\bar{P} \# \bar{P}$.

If $r_{2}=\delta= \pm 1$, then $L_{3,1}=-\varepsilon_{2} \varepsilon_{3} \delta S^{3}$ and $M=W_{1,3} \# W_{3,1}$. The self intersection matrix for $S_{2}$ and $S_{4}$ is

$$
\Lambda=\left(\begin{array}{cc}
\varepsilon_{2} \varepsilon_{3} \delta & 0 \\
0 & -\varepsilon_{4} \varepsilon_{1} \delta
\end{array}\right)=\left(\begin{array}{cc}
\varepsilon_{2} \varepsilon_{3} \delta & 0 \\
0 & \varepsilon_{2} \varepsilon_{3} \delta
\end{array}\right),
$$

and again $M=P \# P$ or $\bar{P} \# \bar{P}$. Note that $r_{3}=2 \delta \varepsilon_{2} \varepsilon_{4}=2 r_{2} \varepsilon_{2} \varepsilon_{4}= \pm 2$ in this case.

If, on the other hand, $\varepsilon_{1} \varepsilon_{4}=\varepsilon_{2} \varepsilon_{3}$, then $\left(m_{2}, n_{2}\right)=-\varepsilon_{1} \varepsilon_{2}\left(m_{4}, n_{4}\right)$ and hence $r_{1}=r_{3}=0$. The two disk bundles $W_{1,3}$ and $W_{3,1}$ are sewn together, as in the case when $r_{2}=0$, so that they give a 2 -sphere bundle over either $S_{2}$ or $S_{4}$. The intersection matrix of $S_{2}$ and $S_{3}$ is

$$
\Lambda=\left(\begin{array}{cc}
\varepsilon_{2} \varepsilon_{3} r_{2} & \varepsilon_{3} \\
\varepsilon_{3} & 0
\end{array}\right) \sim\left(\begin{array}{cc}
\varepsilon_{2} \varepsilon_{3} r_{2} & 1 \\
1 & 0
\end{array}\right) .
$$

If $r_{2}$ is even $M=Q_{0}=S^{2} \times S^{2}$ and if $r_{2}$ is odd $M=Q_{1}=P \# \bar{P}$.

As a summary we have the table:

\begin{tabular}{|c|c|l|}
\hline$t$ & $M$ & \multicolumn{1}{c|}{ Condition } \\
\hline 2 & $S^{4}$ & \\
\hline \multirow{4}{*}{3} & $P$ & $\varepsilon_{1} \varepsilon_{2} \varepsilon_{3}=-1$ \\
\cline { 2 - 3 } & $\bar{P}$ & $\varepsilon_{1} \varepsilon_{2} \varepsilon_{3}=1$ \\
\hline \multirow{4}{*}{4} & $P \# P$ & $\left.\varepsilon_{1} \varepsilon_{4}=-\varepsilon_{2} \varepsilon_{3}\right\} \Rightarrow \begin{array}{c}r_{2}=2 \delta, \varepsilon_{2} \varepsilon_{3} \delta=1\left(\Rightarrow r_{3}=\varepsilon_{3} \varepsilon_{4}\right) \\
\text { or } \\
r_{2}=\delta, \varepsilon_{2} \varepsilon_{3} \delta=1\left(\Rightarrow r_{3}=2 \varepsilon_{3} \varepsilon_{4}\right)\end{array}$ \\
\cline { 2 - 4 } & $\bar{P} \# \bar{P}$ & $\left.\varepsilon_{1} \varepsilon_{4}=-\varepsilon_{2} \varepsilon_{3}\right\} \Rightarrow \begin{array}{c}r_{2}=2 \delta, \varepsilon_{2} \varepsilon_{3} \delta=-1\left(\Rightarrow r_{3}=-\varepsilon_{3} \varepsilon_{4}\right) \\
\text { or } \\
r_{2}=\delta, \varepsilon_{2} \varepsilon_{3} \delta=-1\left(\Rightarrow r_{3}=-2 \varepsilon_{3} \varepsilon_{4}\right)\end{array}$ \\
\cline { 2 - 3 } & $Q_{0}=S^{2} \times S^{2}$ & $\varepsilon_{1} \varepsilon_{4}=\varepsilon_{2} \varepsilon_{3}$, both $r_{2}$ and $r_{3}$ are even (at least one is 0$)$ \\
\cline { 2 - 4 } & $Q_{1}=P \# \bar{P}$ & $\varepsilon_{1} \varepsilon_{4}=\varepsilon_{2} \varepsilon_{3}$, either $r_{2}$ or $r_{3}$ is odd (the other one is 0$)$ \\
\hline
\end{tabular}


Finally, we remark that when $t=4$, unlike $t=2$ and 3 , automorphisms of the group $G$ do not yield all the actions from a given action.

5.7. $t>4$.

THEOREM. If $t>4$, then $M$ is an equivariant connected sum of $P, \bar{P}$ and $Q_{0}$.

Proof. We proceed by showing that if $t>4$, then there exists an $\operatorname{arc} L_{i, j}^{*}$ such that $L_{i, j}= \pm S^{3}$ and hence $M=W_{i, j} \# W_{j, i}$. We use induction and the explicit descriptions above to complete the argument.

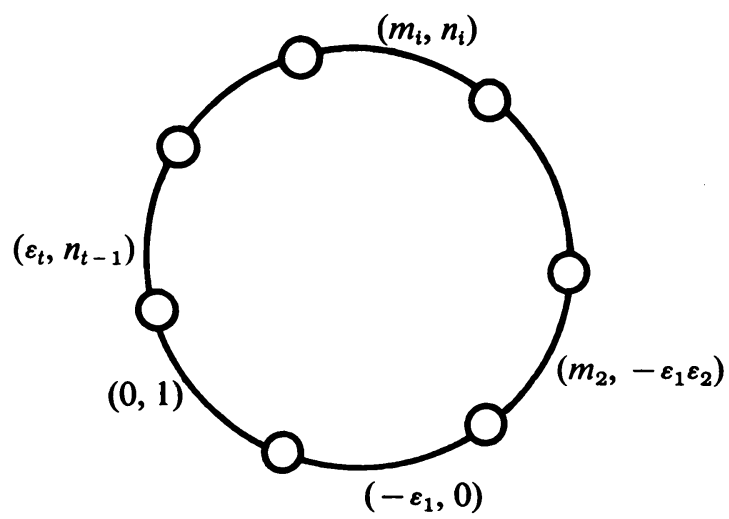

An automorphism, or what is the same a change of parameters, of $G$ alters the action without changing the manifold. Thus we may assume, for convenience, that $\left(m_{t}, n_{t}\right)=(0,1)$ and $\left(m_{1}, n_{1}\right)=\left(-\varepsilon_{1}, 0\right)$. Since

$n_{2}=-\varepsilon_{1} \varepsilon_{2}$ and since

$$
\left|\begin{array}{cc}
-\varepsilon_{1} & m_{2} \\
0 & n_{2}
\end{array}\right|=\varepsilon_{2}
$$

$$
\left|\begin{array}{ll}
m_{t-1} & 0 \\
n_{t-1} & 1
\end{array}\right|=\varepsilon_{t}
$$

$m_{t-1}=\varepsilon_{t}$. Now consider the sequence

$$
\left|\frac{-\varepsilon_{1}}{0}\right|,\left|\frac{m_{2}}{\varepsilon_{1} \varepsilon_{2}}\right|, \ldots,\left|\frac{m_{i}}{n_{i}}\right|, \ldots,\left|\frac{\varepsilon_{t}}{n_{t-1}}\right|,\left|\frac{0}{1}\right|,
$$

where $\left|m_{i} / 0\right|$ means $\infty$.

(i) If $m_{i}=0$, then $\left|n_{i}\right|=1$. Suppose $m_{i}=0$, for $i<t-1$. Then choose $L_{1, i}^{*}$ if $i \neq 2$, and $L_{t-1,2}^{*}$ if $i=2$. In either case $L$ is a 3 -sphere.

(ii) If $n_{i}=0$, then $\left|m_{i}\right|=1$. Suppose $n_{i}=0$, for $i>2$. Then choose $L_{t, i}^{*}$ if $i<t-1$ and $L_{t-1,2}$ if $i=t-1$. Again $L$ is a 3 -sphere.

(iii) If for some $i,\left|m_{i} / n_{i}\right|=1$, then $m_{i}= \pm 1$, and $n_{i}= \pm 1$ and we may choose $L_{t, i}^{*}$, unless $i=t-1$, in which case we choose $L_{1, i}^{*}$. Again $L$ is a 3 -sphere.

(iv) We now show that at least one of the above conditions hold. Suppose not, then there is a first integer $j-1$, such that $\left|m_{j-1} / n_{j-1}\right|>1$ and $\left|m_{j}\right| n_{j} \mid<1$, for $2 \leqq j-1 \leqq t-3$. 
Since

$$
\varepsilon_{j}=\left|\begin{array}{ll}
m_{j-1} & m_{j} \\
n_{j-1} & n_{j}
\end{array}\right|=m_{j-1} n_{j}-m_{j} n_{j-1},
$$

we have $m_{j-1} n_{j}=\varepsilon_{j}+m_{j} n_{j-1}$, and in particular, $\left|m_{j-1}\right|\left|n_{j}\right| \leqq 1+\left|m_{j}\right|\left|n_{j-1}\right|$. But,

$$
\left(\left|n_{j}\right|+1\right)\left(\left|n_{j-1}\right|+1\right) \leqq 1+\left|m_{j}\right|\left|n_{j-1}\right| \text {. }
$$

This yields a contradiction as $\left|n_{j-1}\right|>0$ and $\left|m_{j}\right|>0$. This completes the proof.

Without normalizing, what this actually proved is that for adjacent pairs $\left(m_{i}, n_{i}\right)$ and $\left(m_{i-1}, n_{i-1}\right)$ one can always find a different pair $\left(m_{j}, n_{j}\right)$ equal to either $\pm\left(m_{i}, n_{i}\right), \pm\left(m_{i-1}, n_{i-1}\right)$ or $\left(\varepsilon m_{i} \pm m_{i-1}, \varepsilon n_{i} \pm n_{i-1}\right), t>4$. We wish to thank George Cooke for supplying part of the computation above.

5.8. Remark. The decomposition of $M$ as a connected sum is not unique. In particular the following diagram answers in the affirmative a question of Milnor [3]:

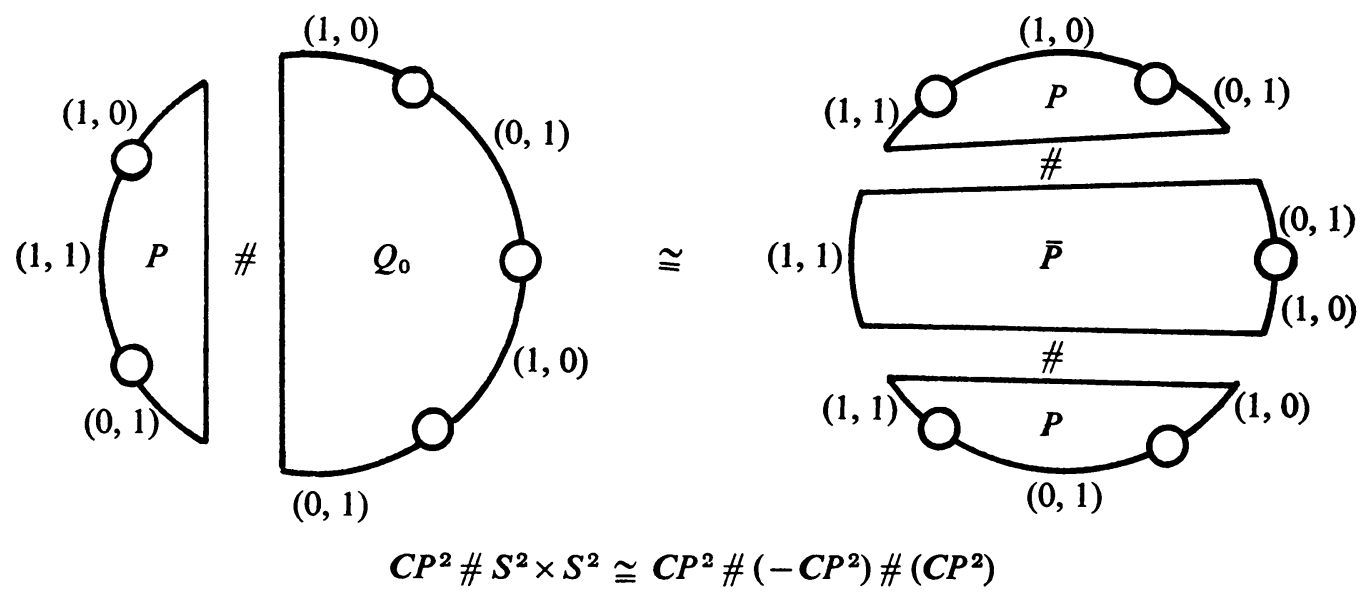

5.9. REMARK. We have already observed that the submanifold $W_{i-1, i+1}$ is a $D^{2}$ bundle over $S_{i}$ with characteristic class

$$
\omega_{i}=\varepsilon_{i} \varepsilon_{i+1}\left|\begin{array}{ll}
m_{i-1} & m_{i+1} \\
n_{i-1} & n_{i+1}
\end{array}\right| .
$$

In general the manifold $W_{i, j}$ is the result of the linear plumbing (in the sense of Hirzebruch [2]) according to the graph

$$
\stackrel{\omega_{i+1} \quad \omega_{i+2} \cdots}{\omega_{j-1}}
$$

5.10. Remark. $P \# \bar{P}=\{(1,1),(1,0),(1,1),(2,3)\}$, is a simple example of an action of a connected group on a manifold that is a connected sum, with the property that there is no invariant 3-sphere separating the components of the connected sum. 
Appendix. Let $X$ be an orientable 4-dimensional cohomology manifold (cm) over the rational numbers $Q$. We shall assume that there exists a closed discrete set $A \subset X$ for which $Y=X-A$ is a cm over $Z$. This set $A$ may be empty. Let the torus $G=S O(2) \times S O(2)$ act effectively on $X$ with a finite number of connected components of orbit types near each point of $A$, and assume $A$ is contained within the fixed point set. (This is a technical assumption which actually can be dropped, however, our arguments would be much more complicated and the conclusions would have to be modified somewhat.)

THEOREM. The orbit space $X^{*}$ is a 2-manifold with boundary. All principal orbits and E-orbits are in the interior and the boundary consists of $C$-orbits and F-orbits. The 4-cm $X$ is locally Euclidean at all points except possibly at $A$. If there are no E-orbits and $F \cup C \neq \varnothing$ then the orbit map has a cross-section.

Proof. Let $x \in X$ be on a 2-dimensional orbit. Choose a slice $S_{x}$. It is necessarily a 2-cm over $Z$ and by Wilder's theorem [10, p. 272] a locally Euclidean 2-manifold. Now $G_{x}$ operates effectively on $S_{x}$ as a finite group of orientation preserving homeomoprhisms and so $S_{x} / G_{x}$, which is a neighborhood of $x^{*}$ in $M^{*}$, is an orientable 2-cm over $Q$ by [7, Theorem 1(i)]. Thus, by Wilder's theorem again, in the neighborhood of $x^{*}, M^{*}$ is an open 2-manifold and consequently by choosing a small closed 2-cell about $x^{*}$ the slice can be cut down to an invariant closed 2-cell whose orbit space is the given closed 2-cell about $x^{*}$. Therefore $G_{x}$, which operates on $S_{x}$, is a cyclic subgroup of $G$ and the action restricted to the invariant closed 2-cell in $S_{x}$ is topologically linear. Observe that if $(G, X)$ was assumed to be a toral action with all orbits two dimensional and $X$ was only assumed to be a 4-cm over $Q$, we would reach the same conclusions as above.

Let us now consider one-dimensional orbits. Again we take a slice $S_{x}$ at $x$ which lies on a 1-dimensional orbit. Let us take a connected component $G_{x}^{0}$ of $G_{x}$. This is a circle subgroup $G(m, n)$ with $(m, n)=1$. It operates effectively on the connected 3 -cm $S_{x}$ over $Z$. All such actions are classified in [8]. In particular, $S_{x}$ is locally Euclidean and the action $\left(G(m, n), S_{x}\right)$ has a fixed point, namely $x$. However, when a circle operates on a 3-manifold the action of the circle is free (locally) away from the fixed point set and the orbit map has a cross-section [8]. Thus $S_{x} / G_{x}^{0}$ is a 2-manifold with boundary corresponding to the image of the fixed points and all other orbits are principal. Choose a closed 2-cell neighborhood about $x^{*}$. Use the cross-section to obtain an invariant (under $G_{x}^{0}$ ) closed 3-cell neighborhood of $x$ in $S_{x}$. It is easy to see that this 3-cell must also be invariant under $G_{x}$ (since $G_{x}=$ $G_{x}^{0} \times G_{x} / G_{x}^{0}$ and the actions commute). On the boundary 2-sphere we find $S O(2) \times H$, where $H \cong G_{x} / G_{x}^{0}$ is a finite subgroup of $G$, acting effectively. But then we can find a group isomorphic to $Z_{p} \times Z_{p}$ acting effectively on the 2 -sphere and preserving orientation. This is impossible, hence $G_{x}^{0}=G_{x}$.

Finally we consider fixed points. Let $x \in F(G, X)$ and $F_{x}(G, X)$ the component of $F(G, X)$ which contains $x$. Let $\left\{G\left(m_{i}, n_{i}\right)\right\}$ be the collection of 1-dimensional 
stability groups for which $F\left(G\left(m_{i}, n_{i}\right), X\right) \cap F_{x}(G, X) \neq \varnothing$. Let $F\left(G\left(m_{i}, n_{i}\right)\right)$ denote the component of $F\left(G\left(m_{i}, n_{i}\right), X\right)$ containing $x$. Put $n_{0}(x)=\operatorname{dim} F_{x}(G, X)$ and $n_{i}(x)=\operatorname{dim} F_{x}\left(G\left(m_{i}, n_{i}\right), X\right)$. If $x \in A$ we have assumed that there are only a finite number of $G\left(m_{i}, n_{i}\right)$, if $x \notin A$, then we can restrict ourselves to a sufficiently small neighborhood of $x$ with compact closure so that at most a finite number of $G\left(m_{i}, n_{i}\right)$ appear. By Borel's formula [1, Chapter XIII, 4.3] we have

$$
4-n_{0}(x)=\sum_{i=1}^{k}\left(n_{i}(x)-n_{0}(x)\right)
$$

where $i$ is summed over all the stability groups $G\left(m_{i}, n_{i}\right)$ for which $F_{x}\left(G\left(m_{i}, n_{i}\right), X\right) \neq \varnothing$. Since $n_{i}(x)>n_{0}(x)$, for $i \geqq 1$, and since $n_{i}(x)=0$ or 2 , and $n_{0}(x)=0$ or 2 , we see immediately that $k=2, n_{0}(x)=0$, and $n_{i}(x)=2$, and $x$ is isolated among the fixed points.

Since $x=F_{x}\left(G\left(m_{1}, n_{1}\right), X\right) \cap F_{x}\left(G\left(m_{2}, n_{2}\right), X\right)$ and both $F_{x}\left(G\left(m_{1}, n_{1}\right), X\right)$ are connected 2-manifolds, the orbit $x^{*}$ is a limit point of both open arcs $\left(F_{x}\left(G\left(m_{i}, n_{i}\right), X\right)\right)^{*}$ on the boundary of the 2-manifold $X^{*}-F^{*}$. We now show that $x^{*}$ fits onto the boundary as one would expect. Since $x$ is isolated from the other fixed points we can assume that $x$ is the only fixed point by choosing a suitably restricted neighborhood of $x$. Thus $X^{*}-x^{*}$ is a 2-manifold with boundary and $x^{*}$ is the one point compactification of a compact neighborhood of $x^{*}$ with $x^{*}$ deleted. Because $X^{*}$ is cohomology locally connected over $Q, x^{*}$ cannot be the limit point of a number of handles from interior of $X^{*}-x^{*}$ nor could it be the limit point of a number of boundary components with stability groups of type $G(m, n)$ other than $F_{x}\left(G\left(m_{1}, n_{1}\right), X\right)$ and $F_{x}\left(G\left(m_{2}, n_{2}\right), X\right)$. Thus, the neighborhood of $x^{*}$ in $X^{*}$ looks like:

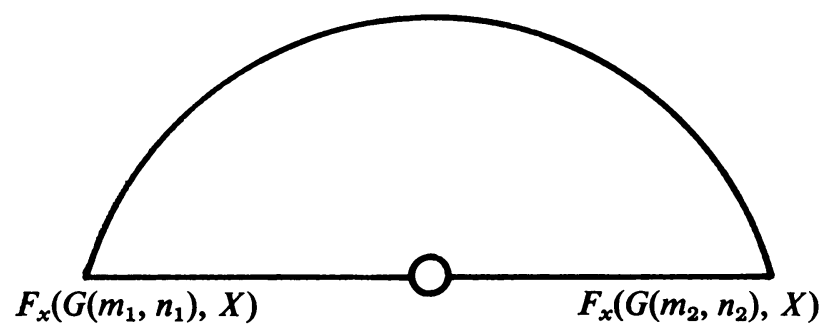

Because $X^{*}$ is Hausdorff the compactification of the deleted neighborhood of $x^{*}$ yields a 2-manifold with boundary. (Actually a more algebraic description of this last procedure can be found in [6].) We can now show that the interior points of $X^{*}$ near $x^{*}$ contain only principal orbits. If $x^{*} \in A$ we have already assumed this and if $x^{*} \in Y$ then in a neighborhood of $x^{*}$ there are at most a finite number of orbit types. If it were possible for $x^{*}$ to be a limit point of $E$-orbits then there would exist an infinite number of orbits with the same finite stability group converging to $x^{*}$. By choosing a prime cyclic subgroup, $Z_{p}$, of this finite subgroup we would obtain a sequence of 2-manifolds with $x^{*}$ as a limit point and hence $F\left(Z_{p}, X\right)$ could not be a cm over $Z_{p}$. 
Notice that unlike the smooth case it is no longer necessary that $G\left(m_{1}, n_{1}\right)$ $\cap G\left(m_{2}, n_{2}\right)$ be the identity. However, certainly $\left|\begin{array}{ll}m_{1} & m_{2} \\ n_{2}\end{array}\right| \neq 0$. It only remains to describe the topology of $X$ near $x \in F$. Since near $x^{*}$ only principal orbits and orbits of type $G\left(m_{1}, n_{1}\right)$ and $G\left(m_{2}, n_{2}\right)$ appear we can choose an $\operatorname{arc} B^{*}$ as illustrated.

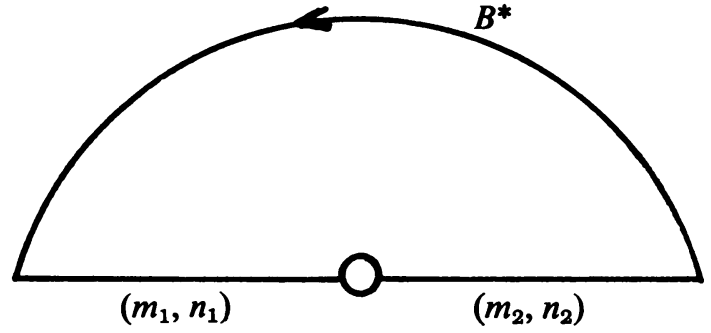

$B=\pi^{-1}\left(B^{*}\right)$ (where $\pi$ is the orbit map) is an invariant lens space $L(r, s)$ with the usual notation of $\S 2$. The arguments of $\S 1$ apply directly and we see that the region bounded by $L(r, s)$ and containing $x$ is just the cone over $L(r, s)$ and the action of $G$ on this cone is (topologically) just the extension of the action of $G$ on $L(r, s)$ to the cone. We see also that $X$ is locally Euclidean at $x^{*}$ if and only if $L(r, s)$ is a 3 -sphere, that is, if and only if $\left|\begin{array}{ll}m_{1} & m_{2} \\ n_{2} & n_{2}\end{array}\right|= \pm 1$. This is also equivalent to saying that $X$ is a 4-cm over $Z$ at $x$. (This is not true for an arbitrary 4-cm over $Z$ but true for one which admits an effective torus action.)

$\$ 1$ now applies for construction of a cross-section under the appropriate hypothesis: Note that

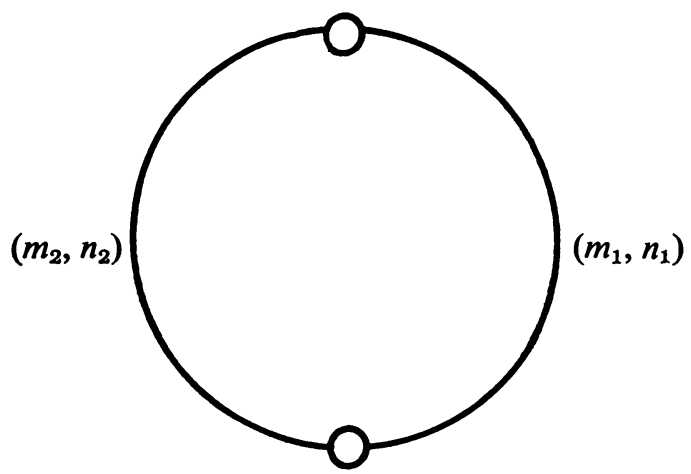

is the suspension of the lens space $L(r, s)$.

This completes the proof of the theorem and also completes the proof of the theorem stated in (1.12).

Remarks. In the compact case when $X^{*}$ is a closed disk, and $E=\varnothing, F \neq \varnothing$, we easily see that $X$ is simply connected. On the other hand, if $H_{1}(X ; Q)=0, X$ is compact, then the arguments of (4.1) imply that $X^{*}$ is a closed 2-disk. If we also assume that $H_{1}(X ; Z)=0$, then an inductive argument rather different from that employed in (4.2) implies that there are no finite isotropy groups. In particular, $X$ would then be simply connected. 
Of course, one has the analogues of the equivariant classification theorem and no doubt the complete analogue of $\$ 5$ can be developed.

EXAMPLE. In order to show that the nonmanifold points introduced above are natural in our context we shall define an action of $T^{2}$ on an algebraic variety $V$ with an isolated singularity. Topologically, $V$ is just a cone over a lens space.

Let $T^{2}$ act on $C^{2}$ by

$$
(\varphi, \theta) \times(x, y) \rightarrow\left(e^{i \varphi} x, e^{i \theta} y\right)
$$

where $0 \leqq \varphi<2 \pi, 0 \leqq \theta<2 \pi$ and $x$ and $y$ are complex numbers.

Define a map

$$
f: C^{2} \rightarrow C^{3}, \quad f(x, y)=\left(i x^{m}-y^{m}, x^{m}-i y^{m},(4 i)^{1 / m} x y\right) .
$$

Note that points in the image $V \subset C^{3}$ satisfy the equation

$$
\left(z_{0}, z_{1}, z_{2}\right) \in V \Leftrightarrow z_{0}^{2}+z_{1}^{2}+z_{2}^{m}=0 .
$$

The point $(0,0,0) \in V$ is an isolated singularity and it is usually called a "quotient singularity" of $C^{2}$. There are $m$ distinct points of $C^{2}$ mapping onto a given point of $V-\{0,0,0\}$.

The $T^{2}$ action defined above induces an action of $T^{2}$ on the variety $V$. Notice that this action is not effective, it has stability group $Z_{m}$ on $V-\{0,0,0\}$. If we divide out by the stability group, we again obtain an action of $T^{2}$ on $V$ and with suitable choice of coordinates for $T^{2}$ the orbit space may be represented as

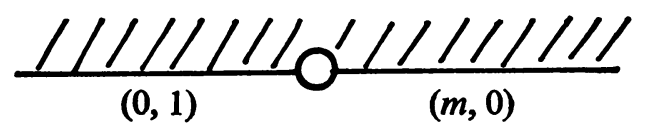

We know from $[4$, p. 80] that $V$ is just the cone over the lens space $L(m, 1)$. From the orbit structure we see that the action is in fact the cone over the action on $L(m, 1)$.

The diagonal action, $\varphi=\theta$,

$$
(\varphi, \varphi)\left(z_{0}, z_{1}, z_{2}\right) \rightarrow\left(e^{i m \varphi} z_{0}, e^{i m \varphi} z_{1}, e^{i 2 \varphi} z_{2}\right)
$$

is the usual $S^{1}$ action on $V$. This circle action is defined on all of $C^{3}$.

We doubt very much that the $T^{2}$ action on $V$ above can be topologically extended to all of $C^{3}$. It certainly cannot be extended algebraically. Furthermore a topological extension that leaves $S^{5}-V$ invariant would imply that $S^{5}-V=$ $S^{5}-L(m, 1)$ fibers over the torus, which is impossible. (It fibers over $S^{1}$ with simply connected fiber [4].)

\section{REFERENCES}

1. A. Borel et al., Seminar on transformation groups, Ann. of Math. Studies, no. 46, Princeton Univ. Press, Princeton, N. J., 1960. MR 22 \#7129.

2. F. Hirzebruch, Differentiable manifolds and quadratic forms, Lecture Notes, Univ. of California, Berkeley, 1962. 
3. J. W. Milnor, On simply connected 4-manifolds, Symposium Internacional de Topología Algebraica, Universidad Nacional Autónoma de Mexico and UNESCO, Mexico City, 1958, pp. 122-128. MR 21 \#2240.

4. J. Milnor, Singular points of complex hypersurfaces, Ann. of Math. Studies, no. 61, Princeton Univ. Press, Princeton, N. J., 1968. MR 39 \#969.

5. P. Orlik and F. Raymond, Actions of $S O(2)$ on 3-manifolds, Proc. Conference on Transformation Groups, Springer-Verlag, Berlin, 1968, pp. 297-318.

6. F. Raymond, The end point compactification of manifolds, Pacific J. Math. 10 (1960), 947-963. MR 22 \#11387.

7. - The orbit spaces of totally disconnected groups of transformations on manifolds, Proc. Amer. Math. Soc. 12 (1961), 1-7. MR 25 \#5127.

8. - Classification of the actions of the circle on 3-manifolds, Trans. Amer. Math. Soc., 131 (1968), 51-78. MR 36 \#2169.

9. R. L. Wilder, Topology of manifolds, Amer. Math. Soc. Colloq. Publ., vol. 32, Amer. Math. Soc., Providence, R. I., 1949. MR 10, 614.

UNIVERSITY OF WISCONSIN,

MadisON, Wisconsin 53706

UNIVERSITY OF MICHIGAN,

Ann Arbor, Michigan 48104 